Draft VERSion JANUARY 1, 2021

Typeset using LATEX twocolumn style in AASTeX63

\title{
Searching for active low-mass stars in CMa star-forming region: multi-band photometry with T80S
}

J. Gregorio-Hetem, ${ }^{1}$ F. Navarete, ${ }^{1}$ A. Hetem, ${ }^{2}$ T. Santos-Silva, ${ }^{1}$ P.A.B. Galli,${ }^{3}$ B. Fernandes,${ }^{1}$ T. Montmerle, ${ }^{4}$ V. Jatenco-Pereira, ${ }^{1}$ M. Borges Fernandes, ${ }^{5}$ H. D. Perottoni, ${ }^{1}$ W. Schoenell,${ }^{6}$ T. Ribeiro, ${ }^{7}$ And A. Kanaan ${ }^{8}$

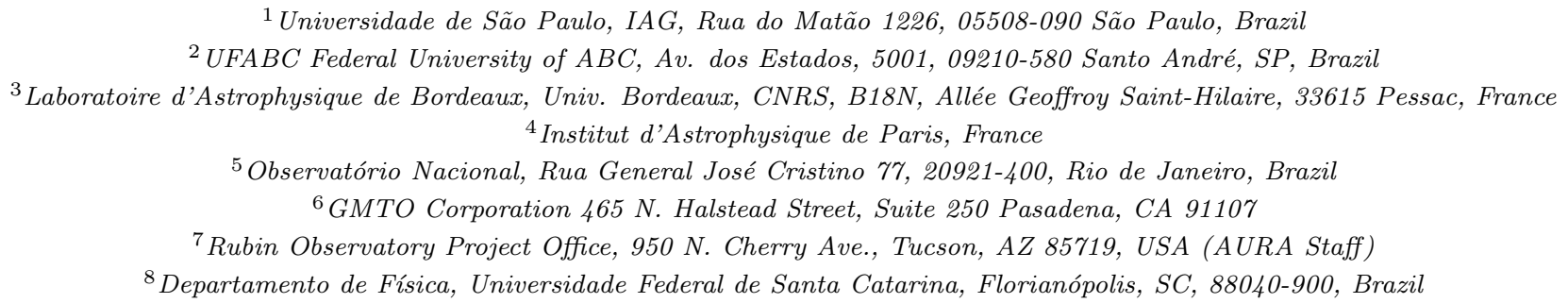

${ }^{1}$ Universidade de São Paulo, IAG, Rua do Matão 1226, 05508-090 São Paulo, Brazil

${ }^{2}$ UFABC Federal University of ABC, Av. dos Estados, 5001, 09210-580 Santo André, SP, Brazil

${ }^{3}$ Laboratoire d'Astrophysique de Bordeaux, Univ. Bordeaux, CNRS, B18N, Allée Geoffroy Saint-Hilaire, 33615 Pessac, France

${ }^{4}$ Institut d'Astrophysique de Paris, France

${ }^{5}$ Observatório Nacional, Rua General José Cristino 77, 20921-400, Rio de Janeiro, Brazil

${ }^{6}$ GMTO Corporation 465 N. Halstead Street, Suite 250 Pasadena, CA 91107

${ }^{7}$ Rubin Observatory Project Office, 950 N. Cherry Ave., Tucson, AZ 85719, USA (AURA Staff)

${ }^{8}$ Departamento de Física, Universidade Federal de Santa Catarina, Florianópolis, SC, 88040-900, Brazil

(Received MM dd, 2020; Revised MM dd, 2020; Accepted MM dd, 2020)

Submitted to AJ

\begin{abstract}
An exotic environment surrounds the young stellar groups associated with the Canis Major (CMa) OB1/R1 region, which probably was formed under feedback from at least three supernova events having occurred a few million years ago. We use astrometric data from the Gaia-DR2 to confirm the membership of the stars in CMa R1, based on proper motion and parallax, which revealed 514 new members and candidates. The mean age of $5 \mathrm{Myr}$ estimated from the color-magnitude diagram characterizes the sources as likely pre-main sequence candidates. In total, a sample of 694 stars detected with the T80-South telescope was analyzed according to different color-color diagrams, which were compared with theoretical colors from evolutionary models, aiming to reveal the objects that exhibit color excess due to accretion processes. Accretion and magnetic activity were also explored on the basis of empirical flux-flux relation, such as $\mathrm{F}_{660}$ and $\mathrm{F}_{861}$ that are related to $\mathrm{H} \alpha$ and Ca II triplet emission, respectively. A low fraction $(\sim 3$ percent $)$ of the sample have $\mathrm{H} \alpha$ excess and other colors expected for stars exhibiting chromospheric activity. The number of Class I and Class II objects, identified by the infrared (WISE) colors, indicates a disk fraction of $\sim 6$ percent, which is lower than the expected for stellar clusters with similar age. A such large sample of objects associated with CMa R1 without evidences of circumstellar accretion can be interpreted as a lack of disk-bearing stars, unusual for young star-forming regions. However, this may be explained as the result of supernova events.
\end{abstract}

Keywords: stars: pre-main sequence - ISM: clouds - open clusters and associations: general.

\section{INTRODUCTION}

Depicting different scenarios of star formation can bring us important clues for answering some of the major problems in astrophysics, mainly related with the early evolution of stars and protoplanetary disks, with implications on planet formation as well as on the structure and evolution of the Galaxy.

Corresponding author: Jane Gregorio-Hetem gregorio-hetem@usp.br
Despite the impressive progress that star formation study has achieved thanks to the advances on multiband observational data analysis, there are still many open questions related to the physical relation between young star clusters and their respective parental clouds.

Our long-term goal is to study the young stellar population in different Galactic regions, and investigate the influence of the environment and feedback from massive stars (ionization, winds, supernovae) in the formation and evolution of star clusters and circumstellar disks. This work is focused on the star-forming region Canis 
Major (CMa) R1 region, aiming to improve the census of the young stellar population.

The CMa OB1/R1 Association is a large $\left(\sim 100 \mathrm{deg}^{2}\right)$ complex of molecular clouds, emission and reflection nebulae showing evidences of star formation induced by supernova explosions. In a search for emission-line stars, Shevchenko et al. (1999) found about two hundred B stars associated with the reflection nebulae that constitute the $\mathrm{CMa} \mathrm{R} 1$ region, located at $d \sim 1 \mathrm{kpc}$ (for a review, see Gregorio-Hetem 2008).

CMa R1 has a mixing of young objects with ages ranging from 1 to $10 \mathrm{Myr}$, distributed on a large scale region (Gregorio-Hetem et al. 2009). Based on X-ray observations in the direction of the Sh 2-296 nebula, SantosSilva et al. (2018) reported more than 300 low-mass young stars candidates. As noted by Fernandes et al. (2015), among the T Tauri stars found by them around Sh 2-296, less than 10 percent show evidence of circumstellar disks. Such low fraction of disk-bearing stars is quite rare when compared with other star-forming regions (Haisch et al. 2001; Hernández et al. 2008; Fedele et al. 2010; Cloutier et al. 2014; Briceño et al. 2019), suggesting that some external factor accelerated the disk dissipation. More recently, Fernandes et al. (2019) identified three runaway stars associated with bow-shock structures in CMa OB1/R1 region. Those authors found that the runaway stars have likely been ejected from three successive SN explosions $(\sim 6, \sim 2$ and $\sim 1 \mathrm{Myr}$ ago), which might have originated the arc-shaped structure named "CMa shell" by them.

In this work we explore the importance of large-scale, multi-wavelength surveys to characterize the pre-main sequence stars associated with CMa R1 in order to investigate aspects such as disk evolution. The goal is to analyze a large reliable sample of members, providing a new optical characterization complemented with the classification based on infrared-excess. By these means, the fractions of accreting stars and disk-bearing stars can be inferred and compared with other stellar clusters with similar age.

The paper is presented as follows. In Sect. 2 we describe the data that we obtained from previous works, and from observations developed by us with the T80South telescope. We also use proper motion and parallax from the Gaia-DR2 catalog to select the sample containing the probable members associated with $\mathrm{CMa}$ R1. The criteria for sample selection and classification are presented in Sect. 3, while the methods based on diagnosis of accretion and magnetic activity, which were adopted to analyze the sample, are described in Sect. 4. Finally, in Sect. 5 we summarize and discuss the main results.

\section{OBSERVATIONS}

\subsection{Data from previous works}

To better understand the star formation scenario and the early evolutionary stages of the stars associated with CMa OB1/R1 we previously analyzed the X-ray sources detected by ROSAT and XMM-Newton satellites in the direction of the nebula Sh 2-296 (Gregorio-Hetem et al. 2009; Santos-Silva et al. 2018). The characterization of the X-ray sources was based on $2 M A S S$ data of their near-infrared counterparts for which mass and age were estimated. This analysis indicates they are probably pre-main sequence stars, but their nature needs to be confirmed by means of complementary optical data.

For a small part (12 percent) of the area where the X-ray sources are found, Fernandes et al. (2015) obtained medium-resolution optical spectra with GMOS (multi-object spectroscopy) at the Gemini telescopes. The GMOS data were used to obtain the spectral classification of the candidates as a diagnosis of their youth nature based on the $\mathrm{H} \alpha$ and lithium lines. The near- to mid-infrared data extracted from $2 M A S S$ and All-WISE catalogues were used to search for evidences of circumstellar structure, which had indicated a low fraction of disk-bearing stars as reported by Fernandes et al. (2015). A similar result was found by Fischer et al. (2016) who adopted the criteria from Koenig \& Leisawitz (2014) for distinguishing Class I, II and III objects that respectively are embedded, disk-bearing, and disk-lacking stars. Fischer et al. (2016) found a few groups of Class I or Class II stars over the entire area of CMa OB1/R1. These groups are shown in Fig. 1, together with the distribution of dust (WISE image) and molecular clouds (CO map) of the region.

Figure 1 also shows the regions covered by different surveys performed by us to study the CMa R1 region: optical spectroscopy with Gemini telescopes (Fernandes et al. 2015), XMM-Newton (Santos-Silva et al. 2018), and optical photometry obtained with T80-South telescope (see Sect. 2.2). In spite of the fact that intermediate-mass stars are well known in this region (Shevchenko et al. 1999; Gregorio-Hetem et al. 2009), the sample of fainter, low-mass stars is still incomplete.

According to the literature, in the area covered by the T80-South observations (red square in Fig. 1), there are 430 stars that are likely associated with the CMa R1 region. Among those previously known objects, 60 corresponds to B- or A-type stars (Claría 1974; Shevchenko et al. 1999) and 370 are young low-mass stars candidates selected from $X M M$ data (Santos-Silva et al. 2018). However, 64 percent of them does not fulfill the parallax 


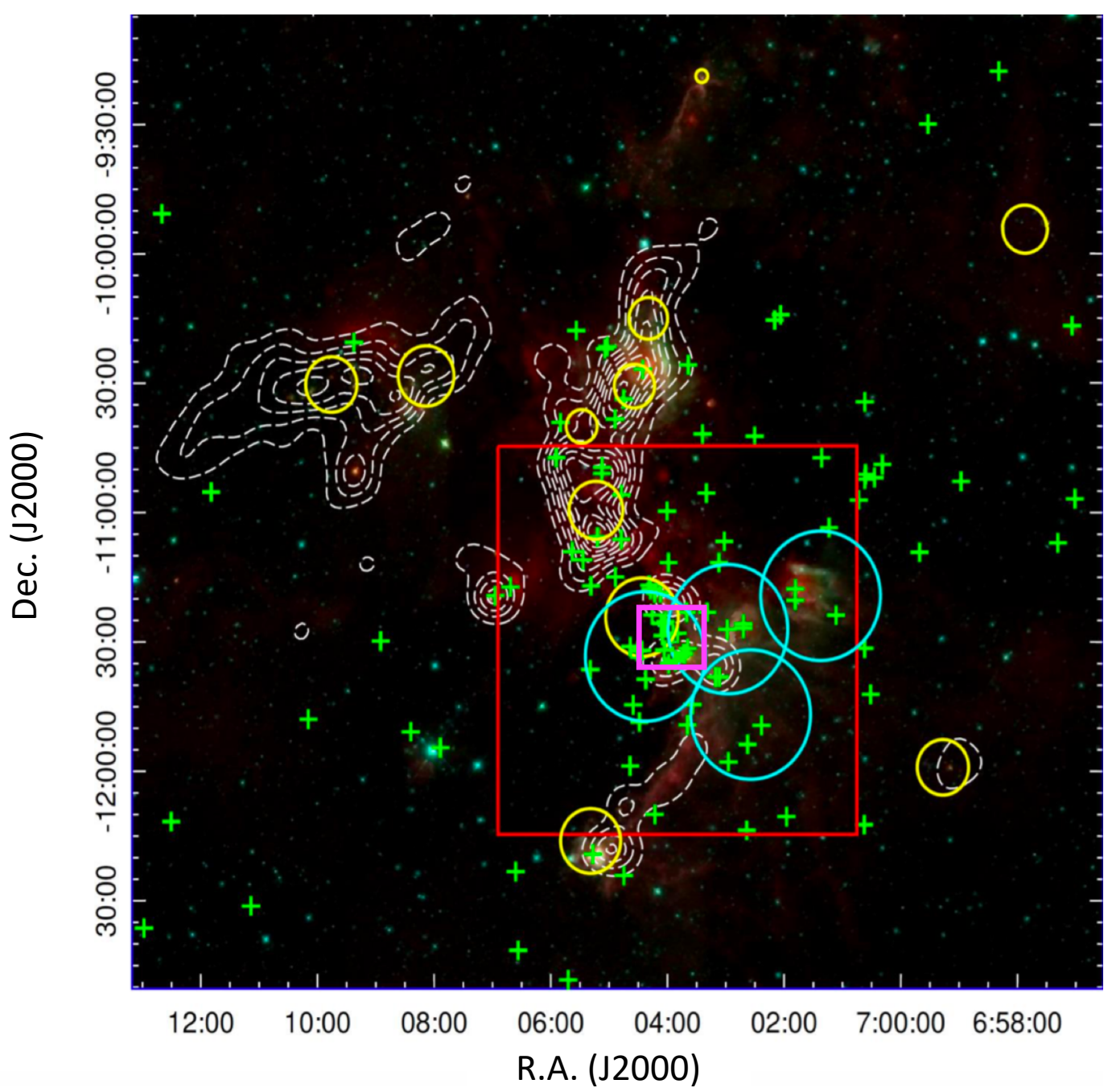

Figure 1. Infrared (WISE, $22 \mu \mathrm{m}$ ) image of CMa R1 region overlapped with ${ }^{13} \mathrm{CO}$ map (dashed contours) from Osaka group (see Onishi et al. 2013), showing the position of B-type stars (green crosses, Shevchenko et al. 1999), and groups of Class I and Class II stars (yellow small circles, Fischer et al. 2016). The fields covered by our observational campaigns are also indicated: T80S (red large box), XMM-Newton (cyan circles), and GMOS (pink small box).

and kinematic criteria used to confirm their association with CMa R1 (see further discussion in Sect. 3.1).

Therefore, the optical observations from T80-South combined with Gaia data analyzed in this work aim both purposes: $(i)$ complementing the characterization of the candidates found by XMM around Sh 2-296, which is necessary to confirm if they are Pre-Main Sequence (PMS) stars, and (ii) seeking the detection of young star candidates in a larger area of the CMa R1 region. Figure 1 (right panel) provides a comparison of the areas covered by our observations showing the distribution of candidates that are X-ray sources $(\sim 0.5 \times 1.3)$, and the confirmed PMS stars, which were observed by GMOS $(\sim 0.23 \times 0.33)$. This illustrates the gain of T80-South observations to identify PMS member candidates across this OB association in a much larger area than the pre- vious follow-up spectroscopic study of Fernandes et al. (2015).

\subsection{Multi-band photometry with T80S}

Optical imaging in a wide $(1.4 \times 1.4)$ field-of-view (FoV), covering the CMa R1 region was performed in 2017, 17 January, with the 0.8-meter telescope T80South (hereafter T80S) located at Cerro Tololo (Chile). T80S is dedicated to performing the "Southern Photometric Local Universe Survey" (S-PLUS) (for more details see Mendes de Oliveira et al. 2019). S-PLUS uses five broadband SDSS filters $\left(u^{\prime}, g^{\prime}, r^{\prime}, i^{\prime}, z^{\prime}\right)$ and seven narrow-band filters centered on features particularly interesting for our study, like Ca II $\mathrm{H}+\mathrm{K}(\lambda=3968.5 \AA$, $3933.7 \AA), \mathrm{H} \alpha(\lambda=6562.8 \AA)$, and Ca II infrared triplet (IRT, $\lambda=8498 \AA, 8542 \AA, 8662 \AA$ ), among others. A set of three images was obtained for each of the 12 filters. 

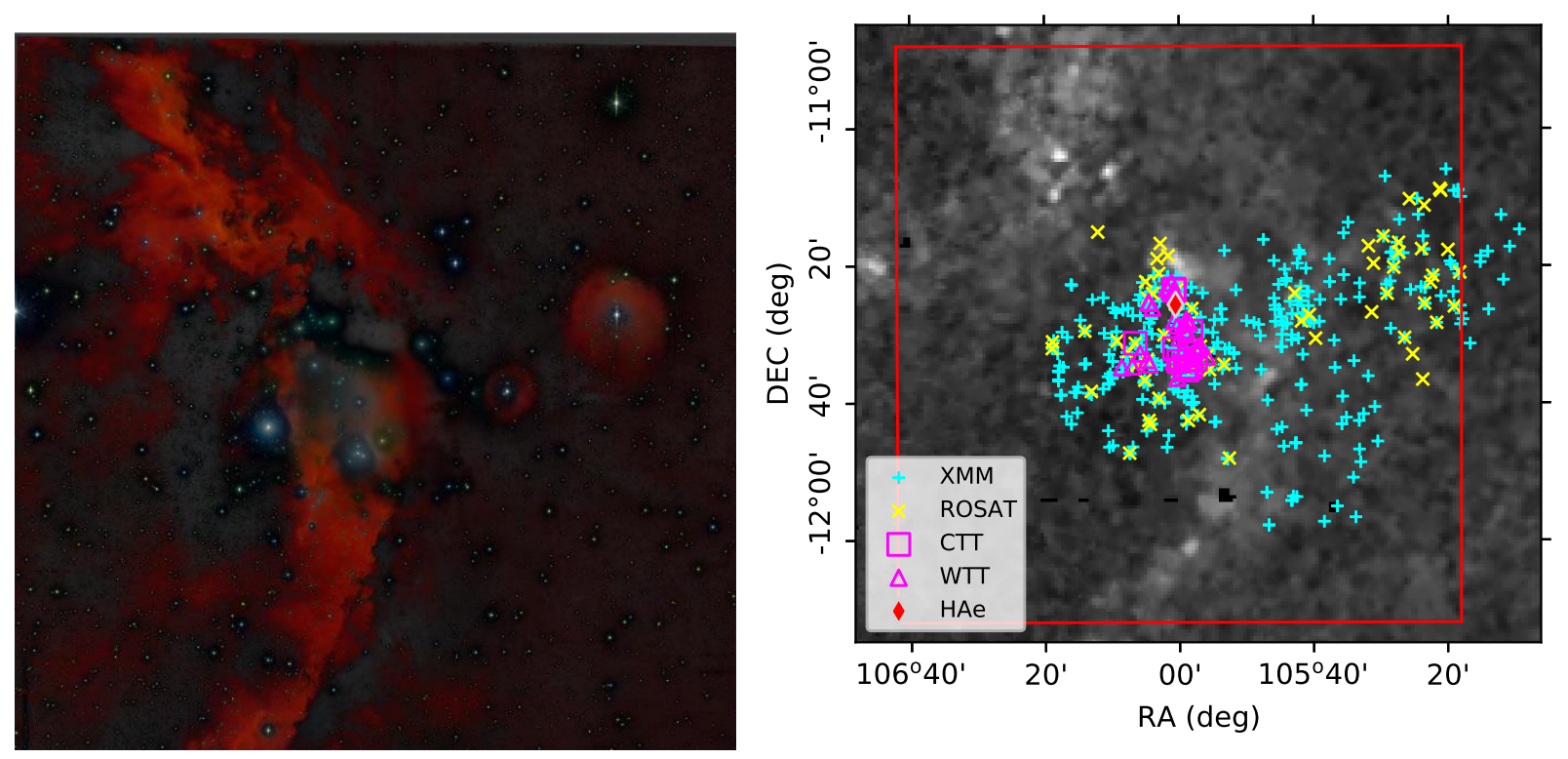

Figure 2. Left panel: Combined image of filters J0660 (H $\alpha$, red), r' (green), and g' (blue) obtained with T80S observations. The field has 1.4 each side. North is up and East is to the left. Right panel: Visual extinction $\left(\mathrm{A}_{V}\right)$ map of CMa R1 overlapped by the T80S FoV (red square), and the position of X-ray sources from Santos-Silva et al. (2018, XMM-Newton) and Gregorio-Hetem et al. (2009, ROSAT). Pink symbols show the previously known PMS stars (Fernandes et al. 2015, GMOS). Inverse scale of colors is used for the map, where dense regions appear in white and light grey indicates low level of extinction $\left(\mathrm{A}_{V}<1\right.$ mag).

Table 2 presents details of data acquisition in the CMa $\mathrm{R} 1$ region.

Figure 1 (left panel) shows a false-color composite RGB image combining three filters, enhancing the nebular $(\mathrm{H} \alpha)$ emission associated with Sh 2-296. For comparison with the dust distribution in the cloud, the right panel of Fig. 1 displays the visual extinction map (Gregorio-Hetem 2008) towards the area covered by $T 80 S$. The position of the X-ray candidates is also shown to indicate the area covered by ROSAT and XMM observations (Gregorio-Hetem et al. 2009; Santos-Silva et al. 2018). Different symbols are used to show were the previously known PMS stars are located.

The data ${ }^{1}$ extraction and calibrations procedures were performed by the S-PLUS team, which developed a pipeline based on the SExtractor software (Bertin \& Arnouts 1996; Bertin 2010) to construct the photometric catalogues (Mendes de Oliveira et al. 2019). The S-PLUS pipeline provides a first estimate of the flux corresponding to the zero-point of magnitude, to be used in the conversion of instrumental magnitudes.

By adopting the mean values of zero-point $\left(\mathrm{ZP}_{o}\right)$ magnitudes estimated by the S-PLUS team ${ }^{2}$ as a first guess, we obtained a preliminary catalogue of about 73700

\footnotetext{
${ }^{1}$ Data reduction is made using the J-PLUS pipeline (Cenarro et al. 2019)

${ }^{2} \mathrm{ZP}_{o}$ calculated by L. San Pedro (private communication).
}

sources extracted from the T80S images. In Sect. 4.1 we present details on the photometric calibration and selection of the sub-sample that is analyzed in this work. The astrometry of the detected sources was checked by comparing their position with the known emission-line stars from Shevchenko et al. (1999).

\section{SAMPLE SELECTION AND CLASSIFICATION}

The main selection criterion is based on the second GAIA data release (Gaia-DR2, Gaia Collaboration et al. 2018a), which was used to confirm the membership of the objects associated with the nebula, and to exclude sources detected by the T80S that are likely field-stars in the line-of-sight of the CMa $\mathrm{R} 1$ region (Sect. 3.1).

Infrared and optical colors were used in order to classify the new PMS members and candidates, as well as the previously known candidates, in comparison with confirmed PMS objects. Firstly we search for sources exhibiting infrared (IR) excess based on data from the WISE survey (Sect. 3.2), and secondly we estimate ages using the Gaia-DR2 (Sect. 3.3).

\subsection{Selection of kinematic members}

The query was restricted to the sources in the parallax range $\varpi=0.8-1.25$ milliarcseconds (mas) that is consistent with the distance of the cloud $(d \sim 1 \mathrm{kpc}$, Gregorio-Hetem 2008). To avoid objects showing low quality of the astrometric solution, we applied the se- 

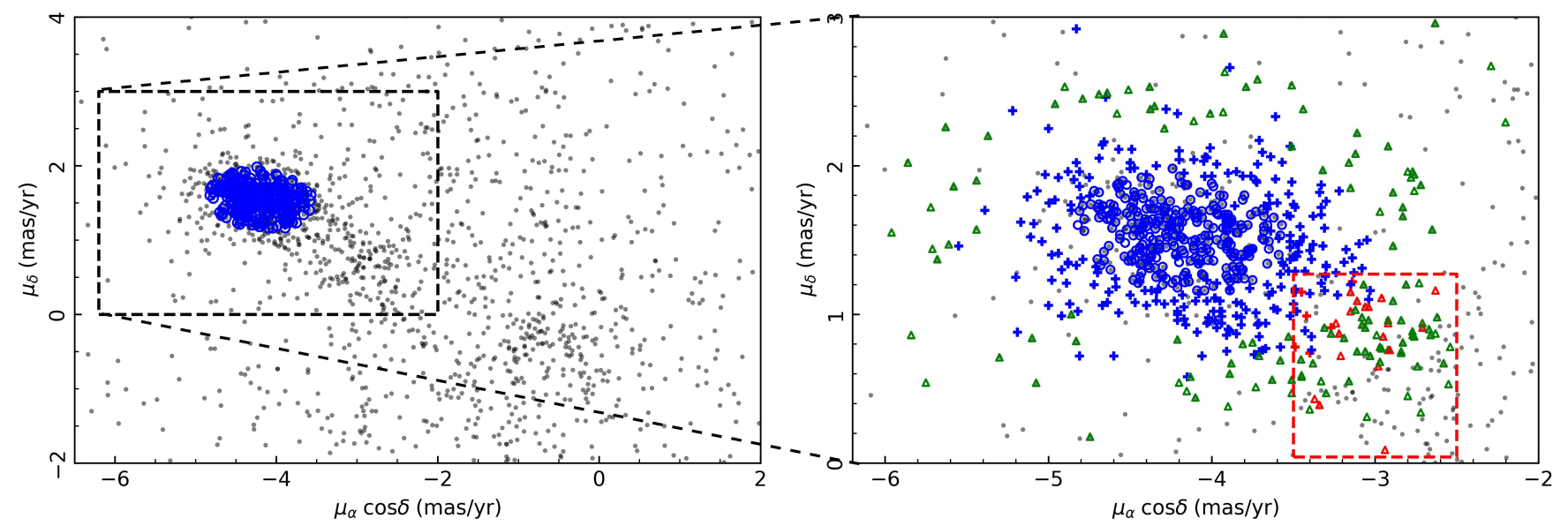

Figure 3. Left: Sources selected from Gaia-DR2 (gray dots) for which we estimate membership probabilities based on proper motion. Blue circles indicate very-likely members with $\mathrm{P} \geq 90$ percent. Right: a zoom of the main clustering of objects observed by both GAIA and T80S surveys. The membership $50 \leq \mathrm{P}<90$ percent defines the probable members (blue + ), while and candidates (green triangles) have $\mathrm{P}<50$ percent. The sources in the apparent sub-cluster (dashed red box) may be part of a group (highlighted by red symbols) that is located to the NW direction of the region (see Fig. 5.)

lection criteria of $\varpi / \sigma_{\varpi}>3$ and $\operatorname{RUWE}^{3}<1.4$ that resulted in an initial list of 4153 objects found in the T80S FoV.

For estimation of membership probability $(\mathrm{P})$ we adopted the method from Hetem \& Gregorio-Hetem (2019) combining a Bayesian model and the Cross Entropy $(\mathrm{CE})$ technique to identify cluster members based on their proper motion $\left(\mu_{\alpha} \cos \delta, \mu_{\delta}\right)$. Due to the high sensitivity of this method to initial conditions, we used a Genetic Algorithm presented by Hetem \& GregorioHetem (2007) to estimate optimized initial values required to create the first generation of parameters for the CE.

The result of the $\mathrm{CE}$ method gives the range of proper motion $\mu_{\alpha} \cos \delta=-4.1 \pm 0.6$ mas $\mathrm{yr}^{-1}, \mu_{\delta}=1.5 \pm$ 0.4 mas $\mathrm{yr}^{-1}$ that was adopted as criterion to select the stars probably associated with the CMa R1 region. According to the proper motion criterion, 78 percent of the objects extracted from Gaia-DR2 probably are field stars $(\mathrm{P}<1$ percent). The remaining list to be studied contains about 900 Gaia sources, 281 of them are verylikely members $(\mathrm{P} \geq 90$ percent), which can be seen in the $\mu_{\alpha} \cos \delta-\mu_{\delta}$ plot presented in Fig. 3 (left panel).

To produce the final list of objects, we performed a cross-matching of the sources selected from Gaia with our preliminary catalog of objects detected with T80S. For the 281 Gaia sources with membership $\mathrm{P} \geq 90$ percent, we have found 208 cross-matching pairs in the $T 80 S$ catalog, meaning that 73 very likely members were

\footnotetext{
${ }^{3}$ Re-normalised unit weight error (see details in the technical note GAIA-C3-TN-LU-LL-124-01)
}

missed in the source extraction performed by the T80S team that uses an automatic pipeline, which is based on aperture photometry. We adopted the Star Finder (SF) procedure (Diolaiti et al. 2000) on three T80S images (filters J0430, J0515, and J0660), seeking for sources that are absent in our preliminary catalog.

The cross-matching of our preliminary catalogue and the list obtained with SF is illustrated in Fig. 4. The magnitudes found by adopting both techniques are in agreement for sources brighter than 20 mag at $660 \mathrm{~nm}$, showing a dispersion of $0.5 \mathrm{mag}$ (or more) in the case of fainter magnitudes. This result gives us confidence in considering in our analysis the list of sources produced by SF, which were missed in the preliminary catalogue. These additional sources were included in our list that contains 669 selected objects: 281 members $(\mathrm{P} \geq 90$ percent), 242 probable members $(50 \leq \mathrm{P}<90$ percent), and 146 candidates $(\mathrm{P}<50$ percent $)$.

Figure 3 (right panel) illustrates our selection criterion that is based on the above mentioned range of proper motion. In the plot centre there is a main clustering where the very-likely members are concentrated (inside an ellipse of $1 \sigma$ size). Around the main clustering we selected the probable members and candidates, which are contained in a $3 \sigma$ distribution that overlaps an apparent sub-cluster (seen to the right). Objects in this sub-cluster may be part of a group located to the NW direction that was identified by Santos-Silva et al. (2020, hereafter SS20) based on a clustering code that uses 5dimensional data from Gaia-DR2 to diagnose physical groups in the direction of the CMa OB1 stellar association. 


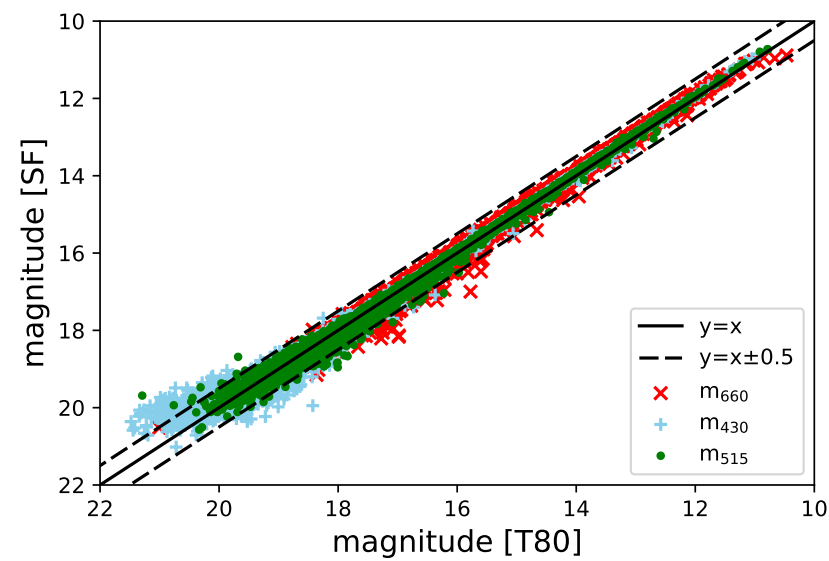

Figure 4. Comparison of magnitudes obtained with filters J0660, J0515 and J0430, by using two different extraction methods: automatic procedure performed by the T80S team and StarFinder (SF) routine adopted by us. A dispersion is found for the faintest sources $\left(m_{430}>20 \mathrm{mag}\right.$.)

Table 1 summarizes the astrometric and kinematic parameters of our sample, compared with the results for two groups found by SS20. The proper motion and parallax of those groups coincide with the range adopted here to select members and candidates: our main clustering has parameters similar to those estimated by SS20 for the group called CMa06, while the sub-cluster seen in Fig. 3 may be part of other group called CMa05.

A view of the distribution of the sample in the equatorial coordinates space is presented in Fig. 5. As expected, it can be noted a clear main concentration of sources at the center, tending to the E direction of the observed region, and roughly following the arc-shaped nebula shown in Fig. 1. According to Gregorio-Hetem et al. (2009), there is a mixing of objects with ages from 1 to $10 \mathrm{Myr}$ in this area that includes the sources at the NW direction. In Sect. 3.3 we revisit these population differences, exploring our sample of young candidate members that is larger than those previously studied.

The list of objects that were previously identified in the literature and were extracted from the Gaia-DR2 according to our selection criteria contains 155 objects. Table 3 gives the kinematic data for 149 of these known objects that were also identified in our T80S catalog. The last lines of Table 3 present the six objects not found in the T80S catalog, probably due to saturation (for bright objects), low $\mathrm{S} / \mathrm{N}$ photometry in the case of faint sources, or nebular contamination.

For comparison purposes, we also analyzed 37 confirmed PMS stars, which were classified by Fernandes et al. (2015) according to $\mathrm{H} \alpha$ and $\mathrm{Li}$ lines detected in GMOS spectra. However, only 6 of them are listed

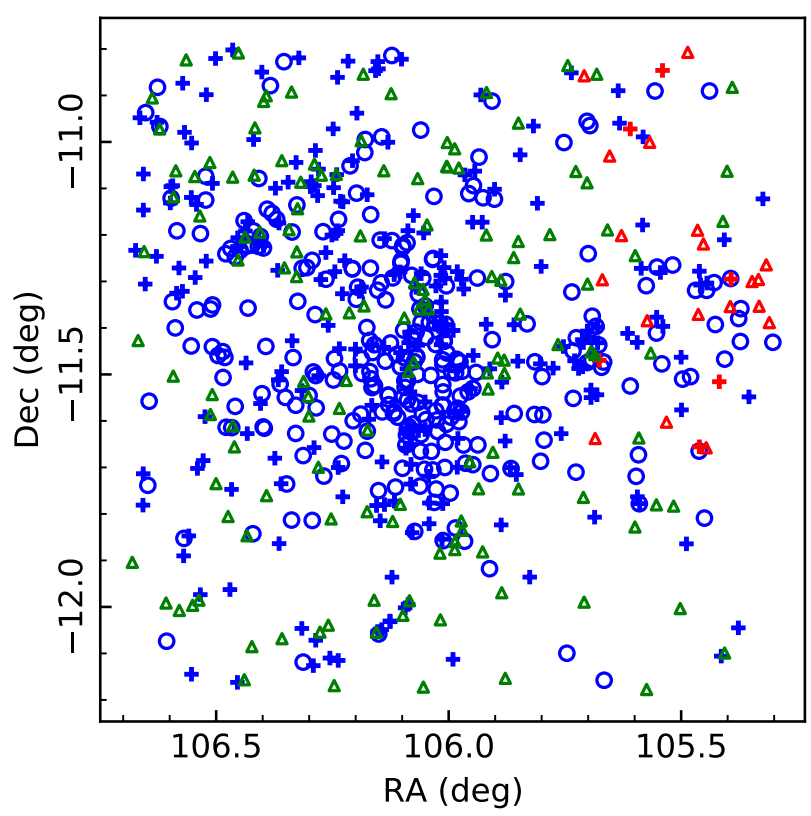

Figure 5. Distribution of candidates and members in the area covered by T80S observations. The symbols are the same used in Fig. 3. In the NW region we use red symbols to highlight the sources appearing in the sub-cluster seen in Fig. 3.

Table 1. Parameters related to the Gaia-DR2 data analysis

\begin{tabular}{cccccc}
\hline \hline ID & RA & Dec & $\varpi$ & $\mu_{\alpha} \cos \delta$ & $\mu_{\delta}$ \\
& J2000 & J2000 & mas & masyr & masyr $^{-1}$ \\
\hline T80S $_{\text {total }}^{a}$ & $106^{\circ} 0 \pm 0.7$ & $-11^{\circ} .5 \pm 0.7$ & $0.8-1.25$ & $-4.1 \pm 0.6$ & $1.5 \pm 0.4$ \\
T80S $_{\text {sub }}^{a, b}$ & $105^{\circ} .5_{-0.2}^{+0.2}$ & $-11^{\circ} 3_{-0.3}^{+0.5}$ & $0.9 \pm 0.1$ & $-3.1 \pm 0.2$ & $0.9 \pm 0.3$ \\
\hline $\mathrm{CMa06}^{c}$ & $106^{\circ} 2 \pm 0.2$ & $-11.4 \pm 0.3$ & $0.85 \pm 0.09$ & $-4.2 \pm 0.4$ & $1.5 \pm 0.2$ \\
$\mathrm{CMa05}^{c}$ & $105^{\circ} 3 \pm 0.2$ & $-11^{\circ} .2 \pm 0.2$ & $0.76 \pm 0.09$ & $-3.0 \pm 0.1$ & $0.8 \pm 0.1$ \\
\hline
\end{tabular}

Note- (a) This work; (b) sub-structure seen in Fig. 3; (c) Results from other work (SS20) that identified two clusters coinciding with our sample.

in Table 3, while the other sources do not fulfill our selection criteria, and/or have poor photometry (mentioned above). To take in account the other 31 known PMS stars that were missing, it was necessary to relax the kinematic criteria in the Gaia data query, and also to adopt a lower threshold limit to extract their T80S fluxes. Table 4 gives the list of these objects: 7 Classical T Tauri stars (CTT), 23 weak-line T Tauri stars (WTT), and one Herbig Ae star (HAe).

In total, our final sample contains 694 objects, which are analyzed in this work. Besides the 180 stars previously identified $(149+31$, above mentioned), there are 514 objects that we suggest to be new young stars associated with CMa R1. Among the new stars identified 
in this work, 395 are probable members $(\mathrm{P} \geq 50$ percent) for which we present the Gaia-DR2 data in Table 5 , while 119 are candidates $(\mathrm{P}<50$ percent $)$ presented in Table 6.

\subsection{Infrared excess}

Part of our sample was previously studied in order to search for disk-bearing stars among the candidates revealed by XMM-Newton data from Santos-Silva et al. (2018), focusing on 205 X-ray sources exhibiting a single IR counterpart (Gregorio-Hetem et al. 2016). The IR colors were compared to the criteria suggested by Koenig \& Leisawitz (2014) to distinguish different PMS classes using WISE photometric bands (Wright et al. 2010), according with the distribution of stars studied in Taurus by Rebull (2010). Only a small fraction (16/205) of our $\mathrm{X}$-ray sources were considered disk-bearing candidates, according to their position in the Class II expected locus. Most $(92 \%)$ of the IR counterparts of the XMM sources in CMa have WISE colors of diskless (Class III) objects.

Here, we extend the same analysis for a larger and more reliable sample in which membership is confirmed by kinematic criteria. Figure 6 shows the $[3.4]-[4.6] \times$ [4.6] - [11.6] diagram of 79 known objects from Table 3, and 357 new members and candidates (Tables 5 and 6) identified with good IR photometric data in the All-WISE Catalog, according with the mitigation and contamination filters proposed by Koenig \& Leisawitz (2014, see their Sect. 2.3). We also plot 26 previously confirmed PMS stars, but in this case, no photometric quality filter was used. Only two objects coincide with the Class I region: a new member, and the Herbig Ae star identified by Fernandes et al. 2015). The Class II locus in Fig. 6 is occupied by six T Tauri stars; 11 known candidates; and 24 new members (Tables 5 and $6)$. In summary, a total of 43 objects were identified in Fig. 6, from which 41 correspond to Class II and two are Class I objects. These sources exhibiting IR excess are highlighted in the following figures.

Taking into account the number of objects with available WISE photometry (463 objects), the fraction of disk-bearing stars (Classe I or II) is 43/463 9 percent. This is an upper limit, since there are other 206 objects of our entire sample that do not have WISE data. It is tempting to suggest these remaining stars probably are Class III, assuming that a lack of data could be due to fainter IR emission. If this hypothesis is correct, the fraction of disk-bearing stars drops to $43 / 694 \sim 6$ percent.

\subsection{Ages}

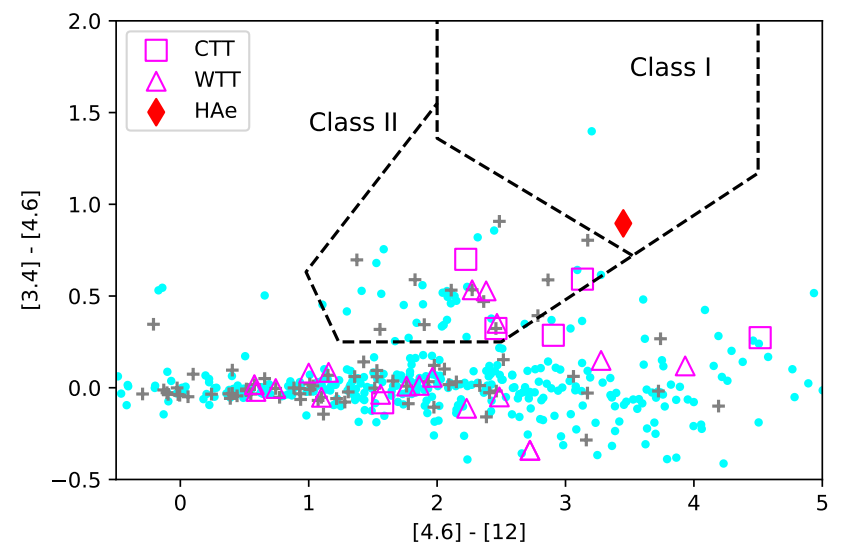

Figure 6. WISE color-color diagram obtained for our sample: new members (cyan dots); previously known candidates $($ grey +$)$; and the T Tauri stars (pink) and one HAe star (red diamont) spectroscopically confirmed by Fernandes et al. (2015). Dashed lines indicate the expected locus of Classes I and II young stars (Koenig \& Leisawitz 2014). The objects outside these boundaries probably are Class III (disk-lacking) stars.

The second step for classifying our sample was to determine the range of the stellar ages using Gaia-DR2 photometry. Figure 7 presents the magnitude-color diagram constructed using the $\mathrm{G}_{\text {Gaia }}$ magnitude as a function of the $\left[\mathrm{G}_{B P}\right]-\left[\mathrm{G}_{R P}\right]$ color. In the same plot, we compare the distribution of the points with isochrones from PARSEC ${ }^{4}$ (Bressan et al. 2012; Marigo et al. 2017). The models for 1 Myr and 5 Myr were adopted to respectively indicate the young- and intermediate-ages, expected for the CMa R1 population (see discussion below). The $100 \mathrm{Myr}$ isochrone was chosen to represent the ZAMS (Zero Age Main Sequence) aiming the comparison with the Main Sequence (MS) colors used by other works discussed in Sect. 4.2.

Most of the T Tauri stars; other previously known objects, and sources exhibiting IR excess (Class II) are $\sim 5$ Myr or younger. About half of the new members and candidates presented in here are in the same range of ages, while the other half are older, but still in the PMS phase.

It is interesting to discuss the spread of ages in comparison with the kinematic characteristics presented in Sect. 3.1. As previously noted by Gregorio-Hetem et al. (2009), CMa R1 has a mixed population. The sources found outside the nebula (to the $\mathrm{W}$ direction) tend to be in the 5-10 Myr range (or older), which can be explained by their location in a region depleted of gas, where the

\footnotetext{
${ }^{4}$ Version v1.2S+COLIBRI PR16 of PARSEC models available on http://stev.oapd.inaf.it/cgi-bin/cmd.
} 


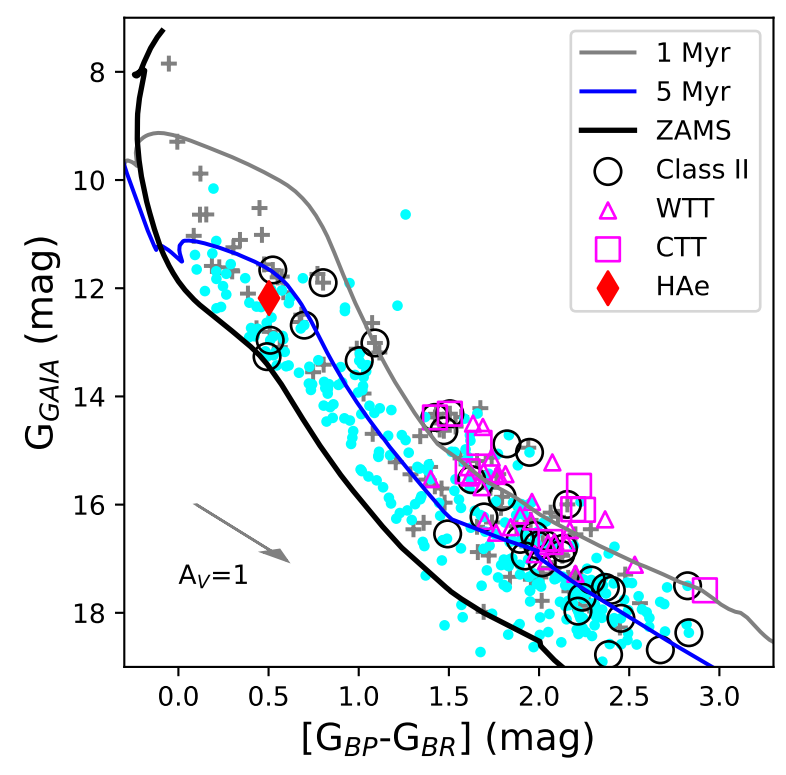

Figure 7. Color-magnitude diagram obtained from GaiaDR2 photometry compared with isochrones from PARSEC. The symbols are the same as Fig. 6, with open circles to highlight the sources exhibiting IR-excess (Class II).

star formation seems to be ceased. This older group coincides with the sub-cluster CMa05 located to the NW direction (see Table 1) for which SS20 estimated an age of $25_{-7}^{+3} \mathrm{Myr}$.

On the other hand, the main clustering associated with the cloud tend to be younger. For the group CM06, which coincides with our main clustering of members, SS20 estimate a mean age of $6_{-1}^{+1}$ Myr. The method adopted by those authors is based on the fitCMD code from Bonatto (2019) that provides accurate age estimates for stellar clusters.

In summary, the age estimation obtained here is validated by other method, giving weight to the characterization of our sample as likely PMS members of CMa R1.

\section{ANALYSIS}

In this section we describe the methodology we adopted to explore the observational data of our targets, by highlighting some of the results from the literature that have inspired this work.

By comparing the photometric data at different bands, we are able to estimate colors of the sources in several spectral domains, which characterize the cloud members. In particular, the flux of $\mathrm{H} \alpha$ and Ca II IRT features combined with $u^{\prime}, g^{\prime}, r^{\prime}$ colors, give us inferences on accretion rate and magnetic activity.

To identify sources showing color excess, it is necessary to refine the ZP magnitudes (mentioned in Sect.
Table 2. Details of the T80S observations.

\begin{tabular}{lccccccc}
\hline \hline filter & $t_{\text {exp }}$ & seeing & $\mathrm{m}_{\text {sat }}$ & $\mathrm{m}_{50 \%}$ & $\lambda$ & $\mathrm{ZP}_{o}$ & $\mathrm{ZP}_{f}$ \\
& $\mathrm{~s}$ & $\prime \prime$ & $\mathrm{mag}$ & $\mathrm{mag}$ & $\AA$ & $\mathrm{mag}$ & $\mathrm{mag}$ \\
\hline${ }^{\prime}$ & 681 & 2.042 & 9.40 & 22.10 & 3574 & $19.64 \pm 0.09$ & 18.80 \\
$\mathrm{~J} 0378$ & 660 & 1.747 & 9.60 & 21.83 & 3771 & $19.07 \pm 0.09$ & 18.55 \\
$\mathrm{~J} 0395$ & 354 & 1.763 & 9.87 & 21.47 & 3941 & $19.09 \pm 0.11$ & 19.00 \\
$\mathrm{~J} 0410$ & 177 & 1.844 & 10.02 & 21.53 & 4094 & $20.10 \pm 0.11$ & 20.20 \\
$\mathrm{~J} 0430$ & 171 & 1.632 & 10.08 & 21.54 & 4292 & $20.26 \pm 0.10$ & 20.45 \\
$g$ & 99 & 1.772 & 10.06 & 21.88 & 4756 & $22.54 \pm 0.09$ & 22.74 \\
$\mathrm{~J} 0515$ & 183 & 1.639 & 9.95 & 21.33 & 5133 & $20.41 \pm 0.08$ & 20.65 \\
$r$ & 120 & 1.617 & 10.00 & 21.12 & 6260 & $22.46 \pm 0.06$ & 22.75 \\
$\mathrm{~J} 0660$ & 870 & 1.775 & 9.96 & 21.02 & 6614 & $19.95 \pm 0.08$ & 20.15 \\
$i$ & 138 & 1.620 & 10.01 & 20.54 & 7692 & $22.30 \pm 0.06$ & 22.44 \\
$\mathrm{~J} 0861$ & 240 & 1.397 & 10.02 & 20.23 & 8611 & $20.58 \pm 0.07$ & 20.70 \\
$z^{\prime}$ & 168 & 1.548 & 10.50 & 20.27 & 8783 & $21.75 \pm 0.06$ & 21.85 \\
\hline
\end{tabular}

NotE- Seeing indicates the quality of photometric observations. The saturation limit is given by $\mathrm{m}_{\text {sat }}$ and photometric depth is $\mathrm{m}_{50 \%}$. Last columns give the first estimate of Zero Point $\left(\mathrm{ZP}_{o}\right)$ and the adopted value $\left(\mathrm{ZP}_{f}\right)$, for which we assume uncertainty of 0.1 mag.

2.2 ) by comparing theoretical data with the observed $\left(u^{\prime}, g^{\prime}, r^{\prime}, i^{\prime}\right.$ and $\mathrm{H} \alpha$ ) color-color diagrams, as presented in the following subsection

\subsection{Calibration}

The data calibration and evaluation of ZP magnitudes were based on spectral energy distribution (SED) fitting and color-color diagram from $g^{\prime}-, r^{\prime}$-, and $i$-band. We analyzed only sources exhibiting well-determined fluxes (signal-to-noise ratio $\mathrm{S} / \mathrm{N}>10$ ) measured with the filter J0660. We also used the photometric depth of the S-PLUS images presented by Mendes de Oliveira et al. (2019), by selecting only sources that are brighter than $21.12 \mathrm{mag}$ for $r^{\prime}$-band, for instance, leading to a list of about 12,000 sources that have good photometric quality. Table 2 gives the $\mathrm{m}_{50 \%}$ photometric depth corresponding to the magnitudes at which 50 percent of the total detected sources are included. On the other hand, the upper limit of saturation is 10 mag at $r$ '-band, which corresponds to the brighter source detected in the T80S image without indication of saturated pixels. In the observed field, there are only four B-type stars brighter than this limit that could be affected by saturation: HD 53396, HD 53456, HD 53457, for which no photometry was extracted from the T80S catalog, and HD 53035 that was detected with $r^{\prime}=9.03 \mathrm{mag}$. Excepting these stars, the sources in our sample are fainter than the saturation limit $\left(\mathrm{m}_{\text {sat }}\right)$ given in Table 2 .

Among the intermediate-mass stars from Shevchenko et al. (1999, SEI99) that were detected by T80S, we selected two to fit their SED: [S99]28 (spectral type B3 ) and [SEI99]160 (HT CMa; A0e), which were used as 


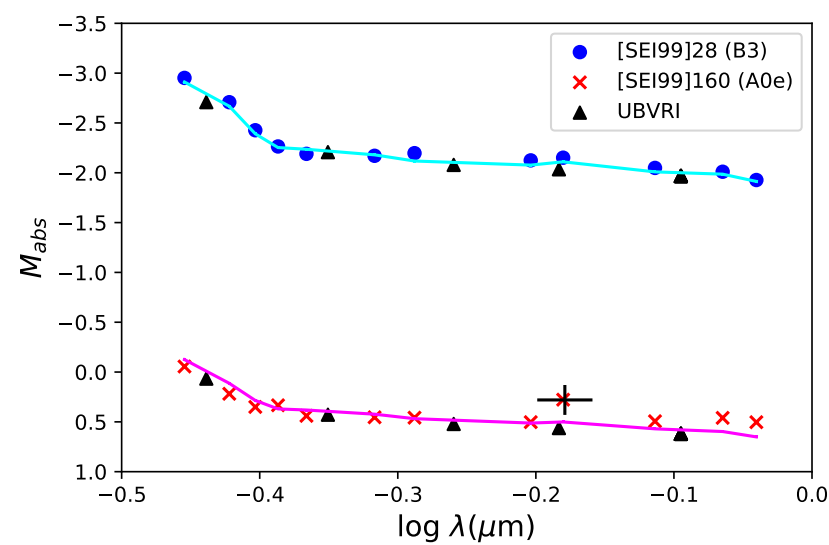

Figure 8. Absolute magnitudes ( $S$ - $P L U S$ photometric system) provided by $P A R S E C$ models (ZAMS) for masses: 5 $\mathrm{M}_{\odot}$ (cyan line), and $3 \mathrm{M}_{\odot}$ (pink line), compared with the T80S photometry obtained for two intermediate-mass stars from Shevchenko et al. (1999, SEI99). The SED fitting is also confirmed by adopting the theoretical colors from the $U B V R I$ system (black triangles) As it is typical for Ae stars, [SEI99]160 shows excess at $\mathrm{H} \alpha$ band, which is indicated by black error-bars.

calibration stars. The SED fitting was obtained by comparison of the observed absolute magnitudes with the theoretical values extracted from PARSEC model for the $100 \mathrm{Myr}$ isochrone that was chosen to represent the ZAMS. Vega magnitudes from two photometric systems were adopted: S-PLUS and UBVRI (Maíz Appellániz 2006; Bessell 1990).

We found for [SEI99]28 a good match with the $5 \mathrm{M}_{\odot}$ stellar model, while the magnitudes of [SEI99]160 are better represented by the $3 \mathrm{M}_{\odot}$ model, excepting for an excess in $\mathrm{H} \alpha$ noted for this star.

Figure 8 shows the theoretical SEDs compared with data of calibration stars. A best fitting was obtained by adopting $\mathrm{A}_{V}=0.3 \mathrm{mag}$ ([SEI99]28) and $1.0 \mathrm{mag}$ ([SEI99]160) for reddening correction of the observed magnitudes. These $\mathrm{A}_{V}$ values agree well with the visual extinction map available for the CMa region (GregorioHetem 2008). Based on the results of the SED fitting, we obtained the final values for $\mathrm{ZP}_{f}$, which are reported in Table 2.

An independent validation of the $\mathrm{ZP}_{f}$ values was obtained by following Mendes de Oliveira et al. (2019, see also Molino et al. 2014) that give the transformations between magnitudes from Gaia-DR2 and T80S, expressed by:

$$
\begin{aligned}
& G_{T 80 S}=+2.0528 m_{660}-1.0594 i^{\prime} \\
& G_{T 80 S}=-0.0372 m_{515}+1.0442 r^{\prime} \\
& G_{T 80 S}=-1.7642 m_{430}+2.7824 g^{\prime}
\end{aligned}
$$

where $i^{\prime}, r^{\prime}, g^{\prime}, \mathrm{m}_{660}, \mathrm{~m}_{515}$, and $\mathrm{m}_{430}$ are the apparent magnitudes measured in the respective bands and calibrated with the $\mathrm{ZP}_{f}$ given in Table 2.

Figure 9a shows the empirical G-band magnitudes $\left(\mathrm{G}_{T 80 S}\right)$ obtained by a linear fit of magnitudes from a set of T80S filters and the Gaia-DR2 G-band magnitudes $\left(\mathrm{G}_{\text {Gaia }}\right)$. A good correlation of $\mathrm{G}_{T 80}$ with $\mathrm{G}_{\text {Gaia }}$ is obtained for most of the sources, which are found in between the dashed lines representing 0.5 mag deviation. The faint sources $\left(\mathrm{G}_{T 80 S}>16 \mathrm{mag}\right)$ show a dispersion, possibly due to different reasons (e.g. variability; photometric errors; etc). Figure $9 \mathrm{~b}$ displays the distribution of mean values of errors as a function of observed magnitudes for each T80S filter, showing that sources fainter than $16 \mathrm{mag}$ have errors larger than 0.05 mag. Bright sources $(\sim 8 \mathrm{mag})$ tend to show larger errors at filters F660 and F861 than those measured in other bands, probably due to nebular contamination combined with saturation effects.

Despite the dispersion of faint sources, the result shown in Fig. 9 can be considered as a validation of the ZP calibration adopted by us. Additional criteria confirming this hypothesis are discussed as follows.

\subsection{Accretion}

Venuti et al. (2014) used $u, g$, and $r$ photometry to estimate accretion rates for the pre-main sequence population of the young cluster NGC 2264. The candidates showing UV-excess were selected from the $[u-g] \times[g-r]$ and $[r] \times[u-r]$ diagrams, where the Classical T Tauri stars (CTTs) occupy a distinguished locus, separated from weak-line T Tauri (WTTs) and field stars.

The $[u-g]$ color is also useful to distinguish candidates showing evidences of UV-excess due to accretion process. For instance, Kalari et al. (2015) obtained $\mathrm{H} \alpha$ and $u$-band accretion rates for CTTs in the Lagoon Nebula M8 by using ugri and $\mathrm{H} \alpha$ photometry from the VST Photometric H $\alpha$ survey (VPHAS+, Drew et al. 2014).

Following Venuti et al. (2014) and Kalari et al. (2015), we present our results in the diagrams shown in Fig. 10, by comparing the observed colors with theoretical MS and ZAMS (PARSEC model, Bressan et al. 2012), whose fitting may validate the $\mathrm{ZP}$ calibration. The previously known candidates exhibiting good quality photometry (see Sect. 4.1) are indicated by grey symbols, while cyan dots are used to show the new members and candidates.

We also highlight in Fig. 10 the distribution of PMS stars characterized on the basis of GMOS spectra (Fernandes et al. 2015).

In Fig. 10a, we compare $\left[r^{\prime}-H \alpha\right] \times\left[r^{\prime}-i^{\prime}\right]$ with MS colors given by Drew et al. (2014). According to Kalari et al. (2015) accreting stars are expected to be 

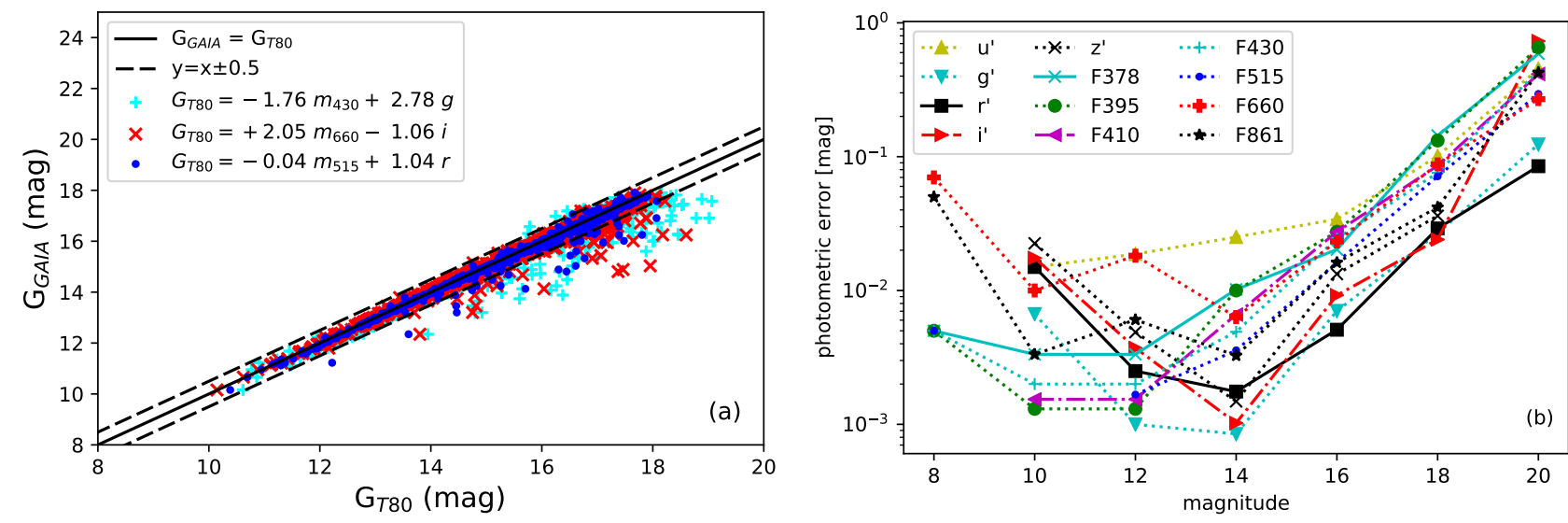

Figure 9. (a) Different relations used to transform T80S photometry into G-band magnitude of the Gaia-DR2 system. (b) Distribution of mean photometric errors as a function of magnitude for each filter, presented in 2 mag wide bins: 7-9, 9-11, etc.

found above the MS line, in a locus where bluer sources $\left(\left[r^{\prime}-i^{\prime}\right]<1 \mathrm{mag}\right)$ have $\left[r^{\prime}-H \alpha\right] \gtrsim 0.5 \mathrm{mag}$, and redder sources have $\left[r^{\prime}-H \alpha\right] \gtrsim 0.8 \mathrm{mag}$. The presence of objects at that region of the plot is interpreted as an evidence of $\mathrm{H} \alpha$ excess related to accretion activity.

Here, we draw attention to 22 objects (marked with error bars) that appear with $\mathrm{H} \alpha$ excess in Fig. 10a: six are known candidates and 16 are new members. Among them there are two Class II stars (black circles, see Sect. 3.2 ), and one Class I candidate (highlighted by a filled circle). However, no $H \alpha$ excess is clearly seen for the other sources having IR excess. Furthermore, none of the confirmed PMS stars can be used as indicator of accretion, since they are found below the MS. This is expected for the WTT stars, but it should not be the case for the HAe and the CTT stars. We tentatively suggest that it can be due to the low $\mathrm{S} / \mathrm{N}$ photometry acquired for these faint sources (see Sect. 3.1).

Among the other candidates previously known that appear in the bottom of Fig. 10a there are two B-type stars listed by Shevchenko et al. (1999): [SEI99]102 and [SEI99]111, for the latter no $\mathrm{H} \alpha$ emission was detected in previous studies (Cohen \& Kuhi 1979; Wiramihardja et al. 1986; Shevchenko et al. 1999) or in the present work. The other stars located at this region of the plot are X-ray sources that could be WTTs, which typically do not exhibit strong $\mathrm{H} \alpha$ emission.

Figure 10b is a color-magnitude diagram comparing the distribution of our sample and the MS line from Covey et al. (2007), by adopting the $\left[g^{\prime}\right] \times\left[g^{\prime}-i^{\prime}\right]$ magnitudes. The data points are also compared with the ZAMS that coincides with the MS in this plot, and the 5 Myr isochrone (PARSEC), representing a mean value of age estimated for the sample (see Fig. 7).

The observed $\left[g^{\prime}-r^{\prime}\right] \times\left[r^{\prime}-i^{\prime}\right]$ colors compared with MS from Covey et al. (2007) are presented in Fig. 10c.
In a similar study, results from Venuti et al. (2014) show no-accreting stars (WTTs) coinciding with the MS, while CTTs are widely scattered around the MS. In our case, only a few objects are found below the MS curve, but we cannot be sure if this is related to accretion or not, since these stars may not exhibit $u^{\prime}$-band excess.

Figure 10d presents the $\left[u^{\prime}-g^{\prime}\right] \times\left[g^{\prime}-r^{\prime}\right]$ diagram used to verify the occurrence of UV-excess in our sample. We also compare our data with the ZAMS. Despite the large dispersion, probably due to low quality of photometry in the $u^{\prime}$-band, most of the objects are likely following the theoretical ZAMS. Only a few stars are found below this curve, which is the expected locus of stars showing UV-excess (Kalari et al. 2015).

By considering only objects of our sample, we suggest that $3.2 \pm 0.7$ percent $(22 / 694)$ seem to have accretion activity as indicated by both $\left[r^{\prime}-H \alpha\right] \times\left[r^{\prime}-i^{\prime}\right]$ and $\left[u^{\prime}-\right.$ $\left.g^{\prime}\right] \times\left[g^{\prime}-r^{\prime}\right]$ diagrams, which confirm our previous results about the lack of disk-bearing stars associated with Sh 2296, which is the main nebula of CMa OB1/R1.

\subsection{Magnectic Activity}

Due to the lack of spectroscopic observations for our sample, we can not evaluate the spectral flux to probe magnetospheric accretion, nor to estimate accretion rates. Alternatively, we can obtain an indirect evidence of activity by using photometry taken at spectral bands containing the aimed spectral lines.

The measurements of $\mathrm{H} \alpha$ flux, combined with the flux of Ca II lines is commonly used as indicators of chromospheric activity in late-type dwarf stars (see references given by Martínez-Arnáiz et al. 2011). The flux-flux relationship between $\mathrm{H} \alpha$ and Ca II IRT $(\lambda 8498 \AA)$ evaluated by those authors shows that young stars have an excess of flux at these bands, when compared with the empirical linear fit found for F- G- and K-type active stars. Despite the differences on the considered wave- 

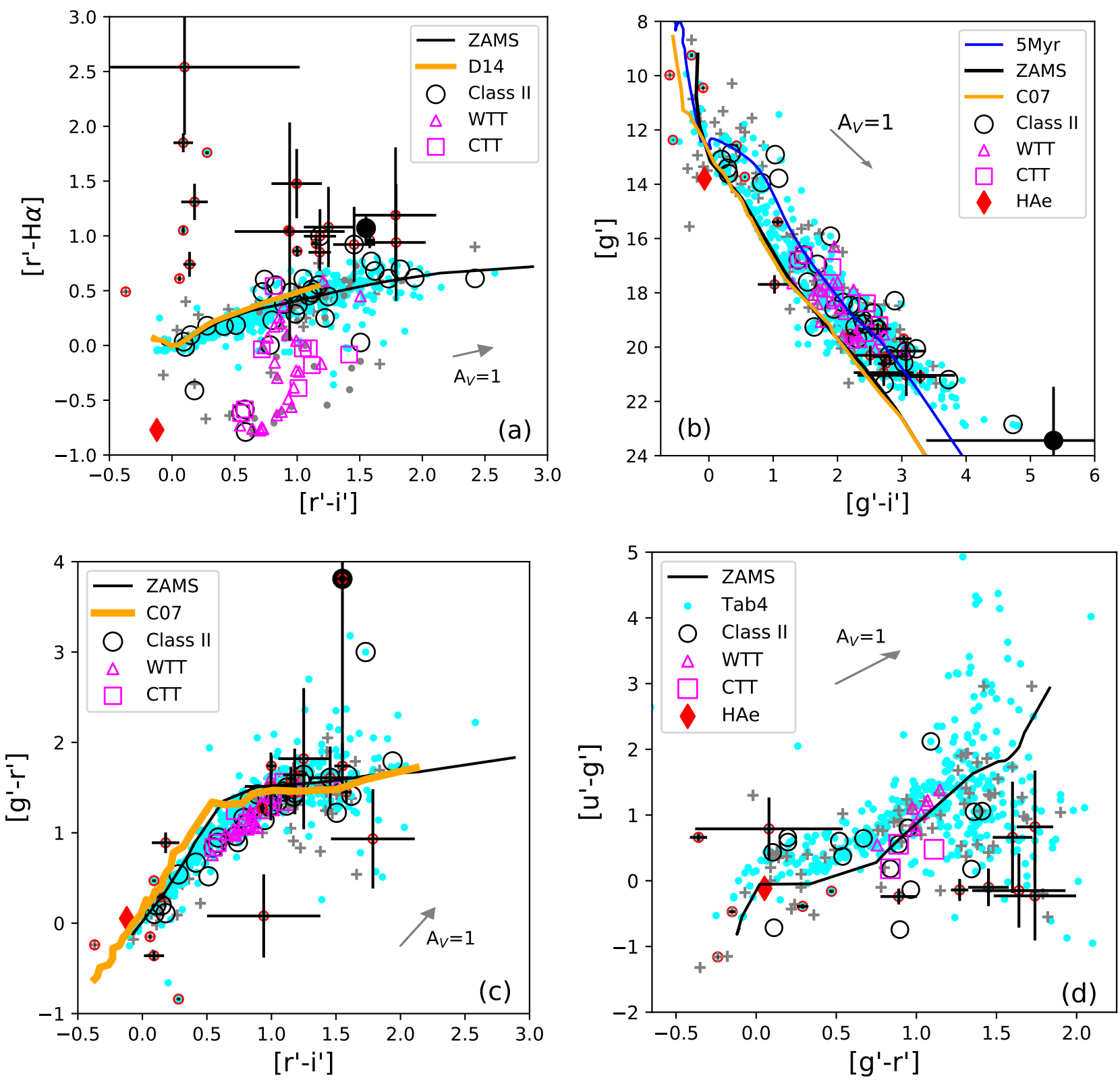

Figure 10. Color-color diagrams obtained for T80S data compared with theoretical colors from PARSEC model (ZAMS, black line), and the Main Sequence (orange line) from Drew et al. (2014, D14), or Covey et al. (2007, C07). The previously known candidates (grey +), and the confirmed PMS members (Fernandes et al. 2015) T Tauri stars (pink squares and triangles) and Herbig Ae star (red diamond) are listed in Table 7. The new members and candidates revealed in this work (cyan dots) are listed in Tables 8 and 9. Open black circles indicate the objects showing WISE colors typical of Class II stars (see Fig. 6), and a filled circle shows one Class I candidate. In panel (a) we note 22 objects exhibiting $\left[r^{\prime}-H \alpha\right]$ color excess, which are marked by red circles with error-bars (for some of them the errors are lower than the symbol size).

length, we used magnitudes measured with filters J0660 and J0861 (see Table 2), aiming to roughly reproduce the flux-flux relationship between $\mathrm{H} \alpha$ and $\mathrm{Ca}$ II IRT, respectively. It is important to note that we are not es- timating line fluxes here, but just comparing broad band fluxes ${ }^{5}$, with no subtraction of continuum.

Figure 11 shows the flux-flux relationship between the fluxes $F_{660}$ and $F_{861}$ observed with $T 80 S$, showing a few

\footnotetext{
5 The conversion of instrumental magnitudes $\left(\mathrm{m}_{\lambda}\right)$ to flux $\left(\mathrm{F}_{\lambda}\right)$ is given by $F_{\lambda}(J y)=3631 \times 10^{-0.4\left[m_{\lambda}+\left(\mathrm{ZP}_{f}\right)_{\lambda}\right]}$
} 


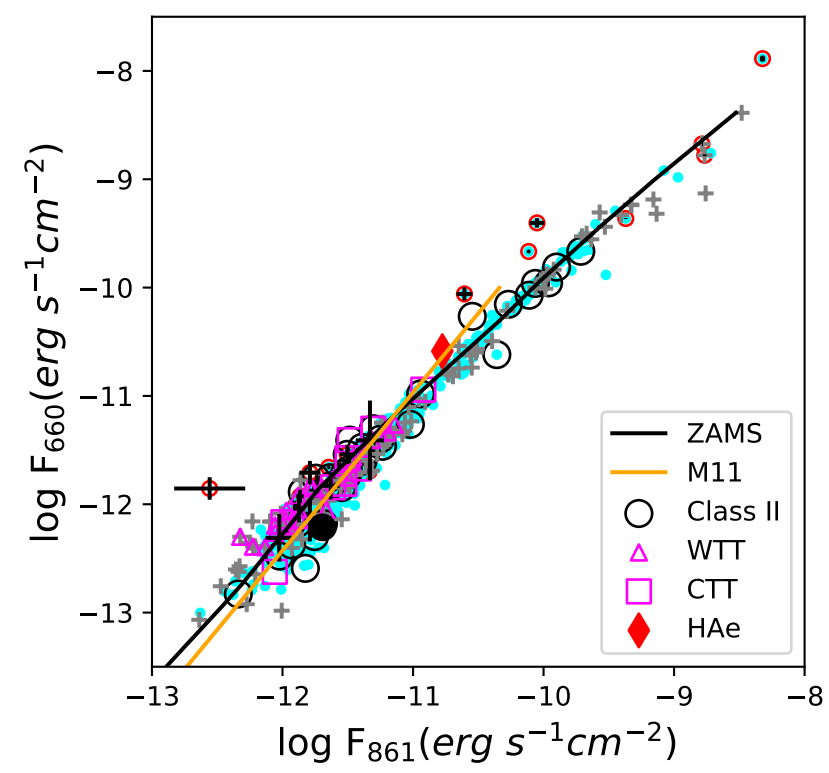

Figure 11. Broad band fluxes associated with the $\mathrm{H} \alpha$ and Ca triplet emission, respectively estimated from the $\mathrm{m}_{660}$ and $\mathrm{m}_{861}$ magnitudes observed with T80S. The flux-flux relation is comparable with results from Martínez-Arnáiz et al. (2011, M11), where active late-type stars are expected to appear above the MS line. Symbols are the same as Fig. 10.

sources above the MS line that could indicate $\mathrm{H} \alpha$ excess. This is not unexpected since most of the objects studied by Fernandes et al. (2015) are WTTs, and the A- and B-emission stars (Shevchenko et al. 1999) present in our sample are not strong $\mathrm{H} \alpha$ emitters.

\section{CONCLUSIONS}

\subsection{Summary of results}

We used the Gaia-DR2 astrometric data to evaluate the membership probability $(\mathrm{P})$ and identify the $\mathrm{CMa}$ R1 members based on proper motion. From Gaia-DR2 we extracted only the objects showing parallax $\varpi=0.8$ - 1.25 mas, compatible with the distance of the cloud (d $\sim 1 \mathrm{kpc}$, Gregorio-Hetem 2008), RUWE $<1.4$ and $\varpi / \sigma_{\varpi}>3$ resulting in a list of $\sim 4,200$ objects in the area observed with $T 80 S$.

Our T80S observations covered a $\sim 2 \mathrm{deg}^{2}$ area around Sh 2-296, the main nebula of the CMa R1 region, where more than 70 thousand objects were detected, 17 percent of them exhibiting good quality photometry.

We selected 669 sources on the basis of membership probability that was estimated with the Bayesian technique. The mean values of proper motion for 523 probable members $(\mathrm{P} \geq 50$ percent $)$ are: $\left(\mu_{\alpha} \cos \delta \sim-4.1 \pm 0.6\right.$, $\left.\mu_{\delta} \sim 1.5 \pm 0.4\right)$ mas $\mathrm{yr}^{-1}$. We also considered 146 candidates ( $\mathrm{P}<50$ percent) exhibiting proper motion compatible within a $3 \sigma$-interval from the mean values. The other objects extracted from Gaia are very likely fieldstars (membership probability: $\mathrm{P}<1$ percent).

Table 3 contains 155 stars that are candidates previously identified in the literature, but only 149 were included in our final sample because six sources were not detected by T80S. Other 31 previously known objects included in our sample are spectroscopically confirmed PMS stars (Table 4), but they have low S/N photometry. In total, our final sample contains 694 sources, 180 of them are previously known objects. Therefore, the membership analysis based on Gaia-DR2 data revealed 395 new members (not previously identified in the literature) and 119 new candidates. Table 5 and Table 6 give the kinematic data and identification of these new members and candidates, respectively.

The color-magnitude diagram $\mathrm{G}_{\text {Gaia }} \times\left[G_{B P}-G_{R P}\right]$ was used to estimate the age of the sources. A mean value of $5 \mathrm{Myr}$ was found, which characterizes our sample as likely PMS members of CMaR1. The presence of IR-excess was investigated on the basis of WISE colors that revealed 43 objects: 2 are Class I and 42 are Class II. Only six of the T Tauri stars have IR-colors typical of Class II (none is Class I), which indicates that most of the confirmed PMS stars of our sample are Class III, exhibiting low IR emission.

The photometric zero-points (ZPs) and calibration of the T80S data were obtained by using different methods (see Sect. 4.1). First, we performed the SED fitting of two stars with well determined spectral type, by adopting PARSEC evolutionary models.

The ZPs were also evaluated by analyzing the $g^{\prime}, r^{\prime}, i^{\prime}$ colors diagram and correlations between the G-band magnitude from Gaia-DR2 $\left(G_{\text {Gaia }}\right)$ and empirical values obtained from T80S data $\left(G_{T 80}\right)$.

Table 7 gives the photometric data for previously known objects that were observed by T80S. The photometric data for the new members and candidates are given in Tables 8 and 9, respectively.

Color-color diagrams were used to confirm the occurrence of CMa R1 members showing color excess related to accretion process, which did not reveal more than 22 stars with excess in the $\mathrm{H} \alpha$ or the $u$ '-band.

Magnetic activity was investigated according the fluxflux relations used by Martínez-Arnáiz et al. (2011) comparing $\mathrm{H} \alpha$ and $\mathrm{Ca}$ II $\mathrm{H} \& \mathrm{~K}$ lines fluxes typically found for late-type stars showing chromospheric activity. An excellent correlation between the broad band $\mathrm{F}_{660}$ and $\mathrm{F}_{861}$ fluxes is found, but only a few stars have indications of $\mathrm{H} \alpha$ excess likely due to accretion.

\subsection{Concluding remarks}


Previously we identified a few hundred young stars candidates associated with the nebulae in CMa R1, but our studies were restricted to small areas, such as the fields covered by XMM-Newton observations. Aiming to explore a more complete and reliable sample of candidates, the Gaia-DR2 catalog was used to select the kinematic members, for which we analyzed multi-band T80S imaging observations that provided very good indicators of accretion activity.

Despite a large sample of new PMS candidates were identified, a small fraction of them show signs of accretion processes and/or magnetic activity. Only $3.2 \pm 0.7$ percent of our sample seem to have $\mathrm{H} \alpha$ and/or UV excess, which gives an estimation of accreting fraction.

Based on the number of Classes I and II sources $(43 / 694)$ we estimate a disk fraction of $6.2 \pm 0.9$ percent. We have noticed in our sample the presence of sources that may be part of a sub-group, which was identified in the NW direction of the region. But none of these sources have IR-excess. Therefore, the number of diskbearing stars is evaluated here for the whole population.

Comparing CMa R1 with other regions, we note that our results are lower than the values expected for young stellar groups with similar ages $(\sim 5 \mathrm{Myr})$. For instance, a disk fraction of more than 10 percent is reported for NGC 2362 (Haisch et al. 2001); and 20 percent for Upper Sco (Fedele et al. 2010; Cloutier et al. 2014). The accretion fraction estimated for these regions is $\sim 5$ percent (Fedele et al. 2010). More recently, Briceño et al. (2019) presented the decline of the accreting fraction, as well as the percentage of disk-bearing stars, as a function of the mean age of each sub-group of the Orion OB1 star-forming region. For Orion $1 b$ sub-group that is 5 Myr old, they report an accreting fraction of about 10 percent, and a disk fraction of $\sim 15$ percent.

We conclude that analyzing the optical photometric data gave us a confirmation of our previous results, which were based on spectroscopy and near-infrared data. Despite the use of a much large sample of candidates, the absence of disk-bearing stars in the region is confirmed. Somehow, circumstellar accretion has finished for the young stars in this region, probably due to the star formation scenario where at least three supernova events occurred a few millions years ago. This could be the cause of the disks disappearance earlier than usually is found in other star-forming regions at same age. 


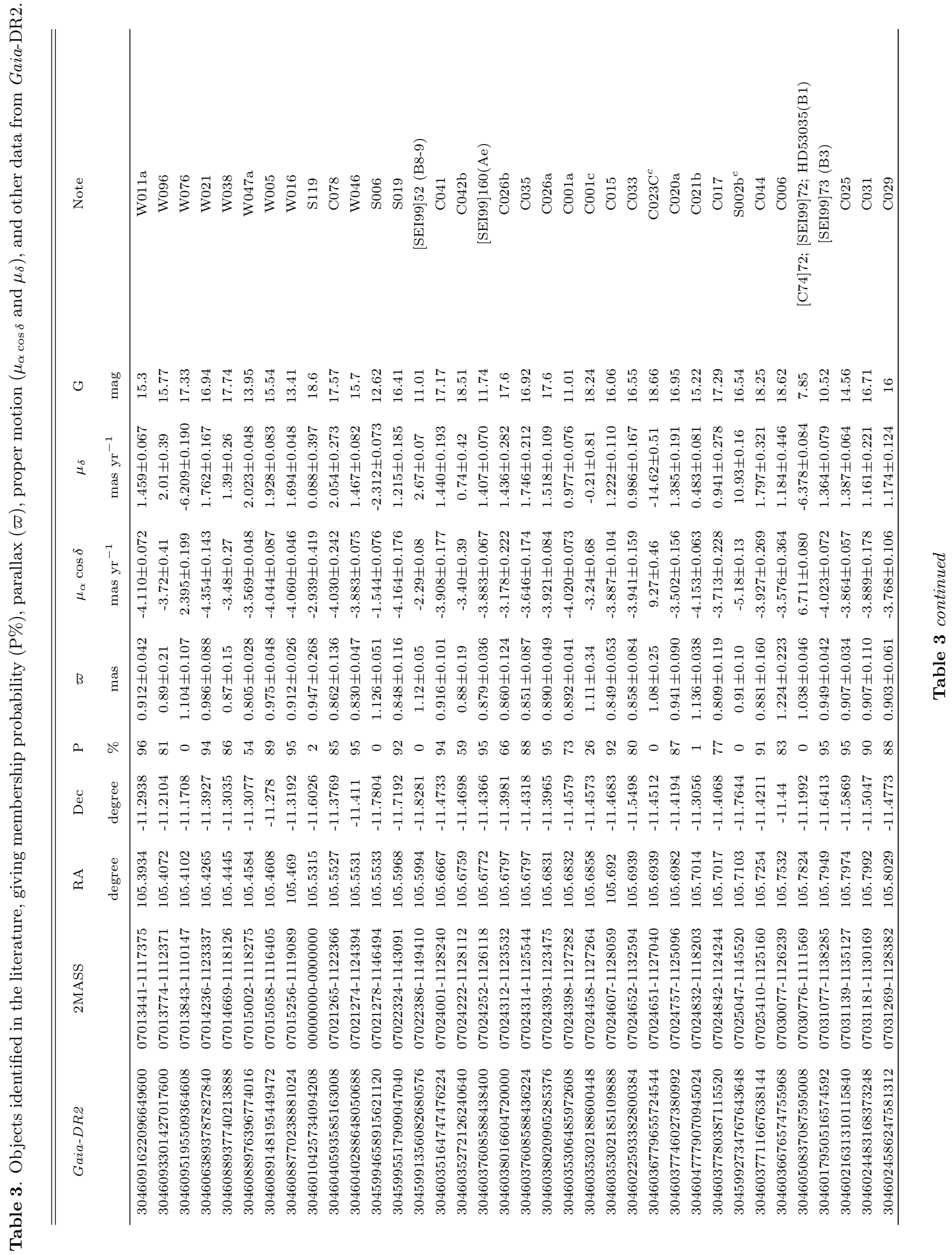




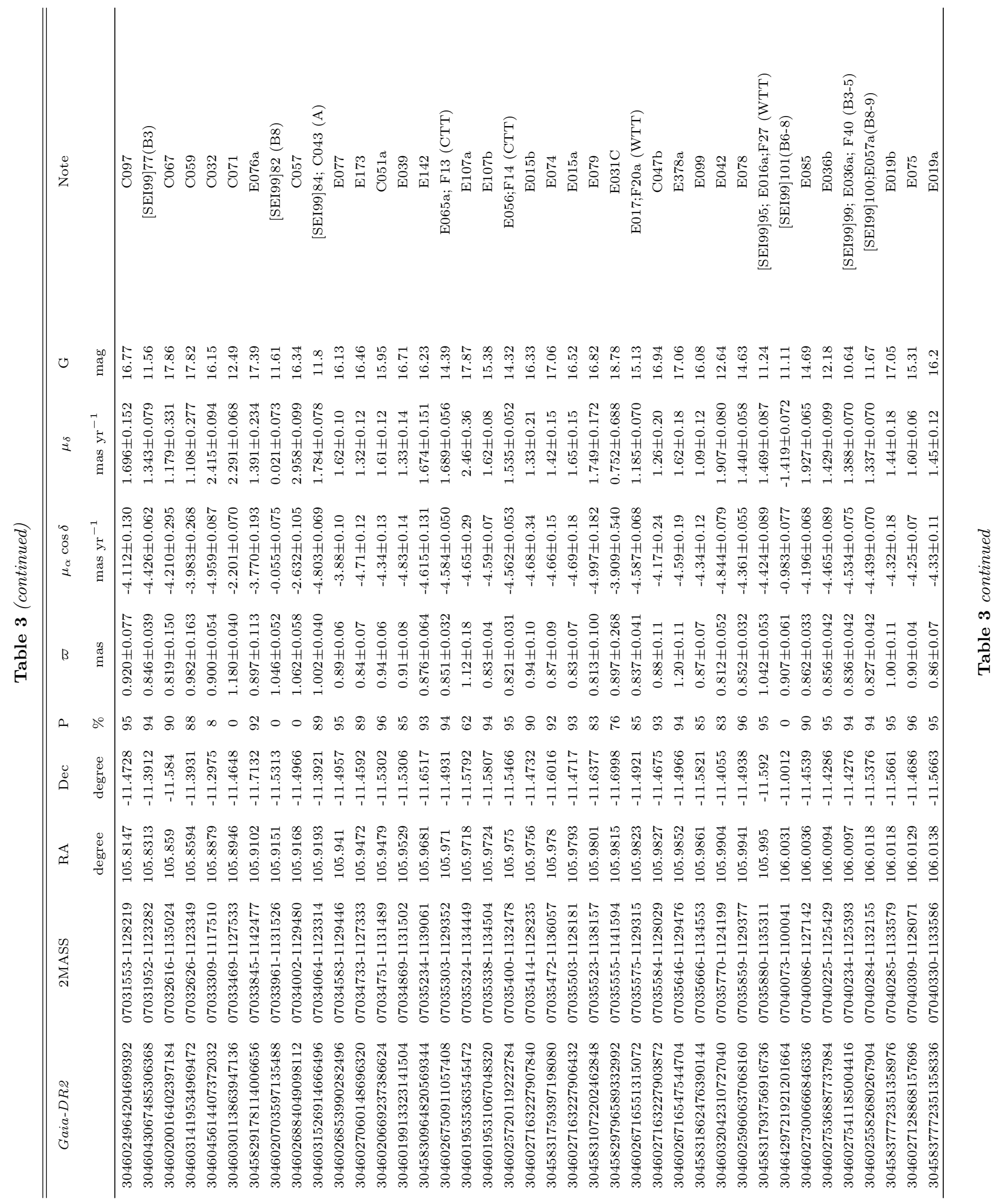




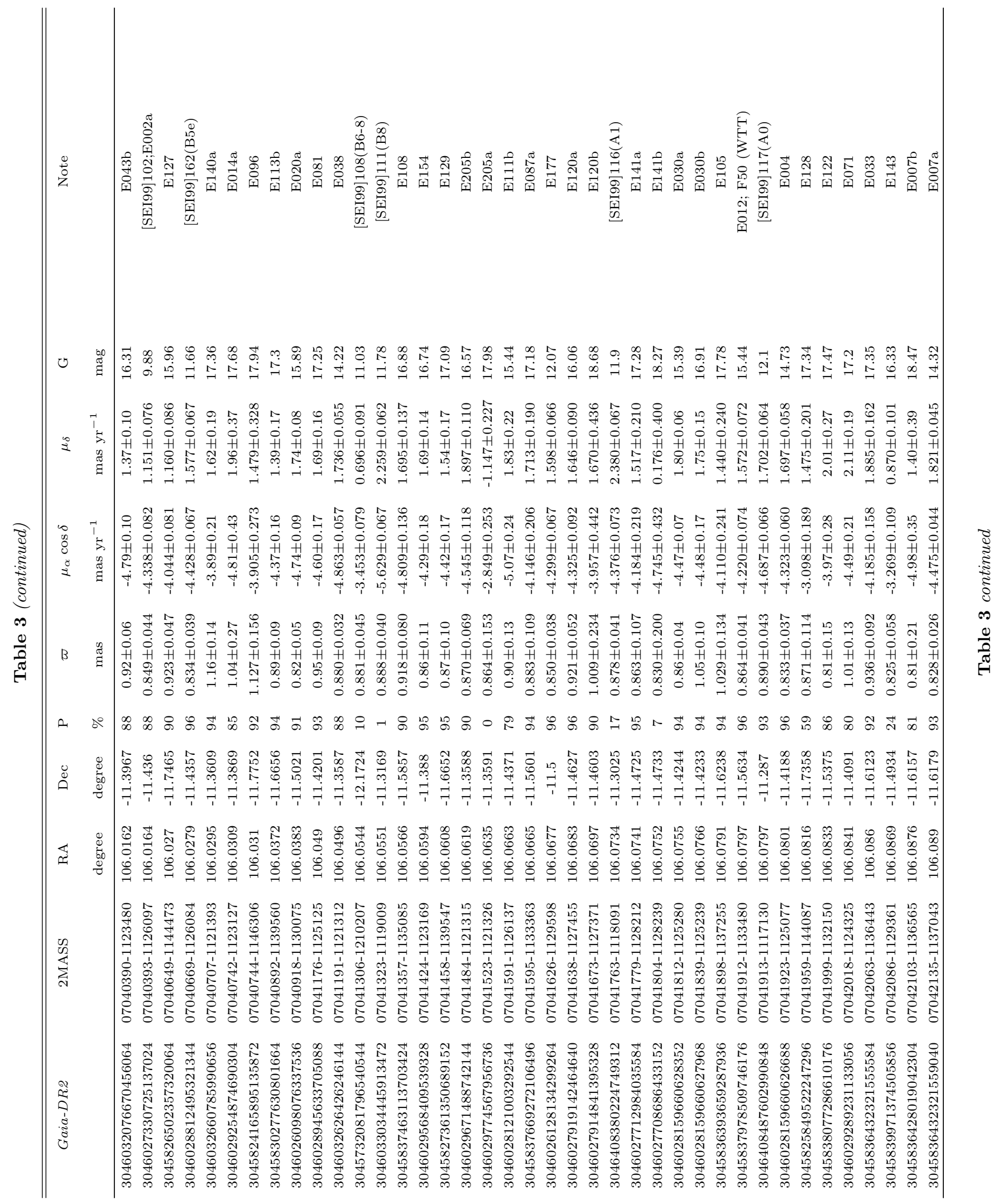




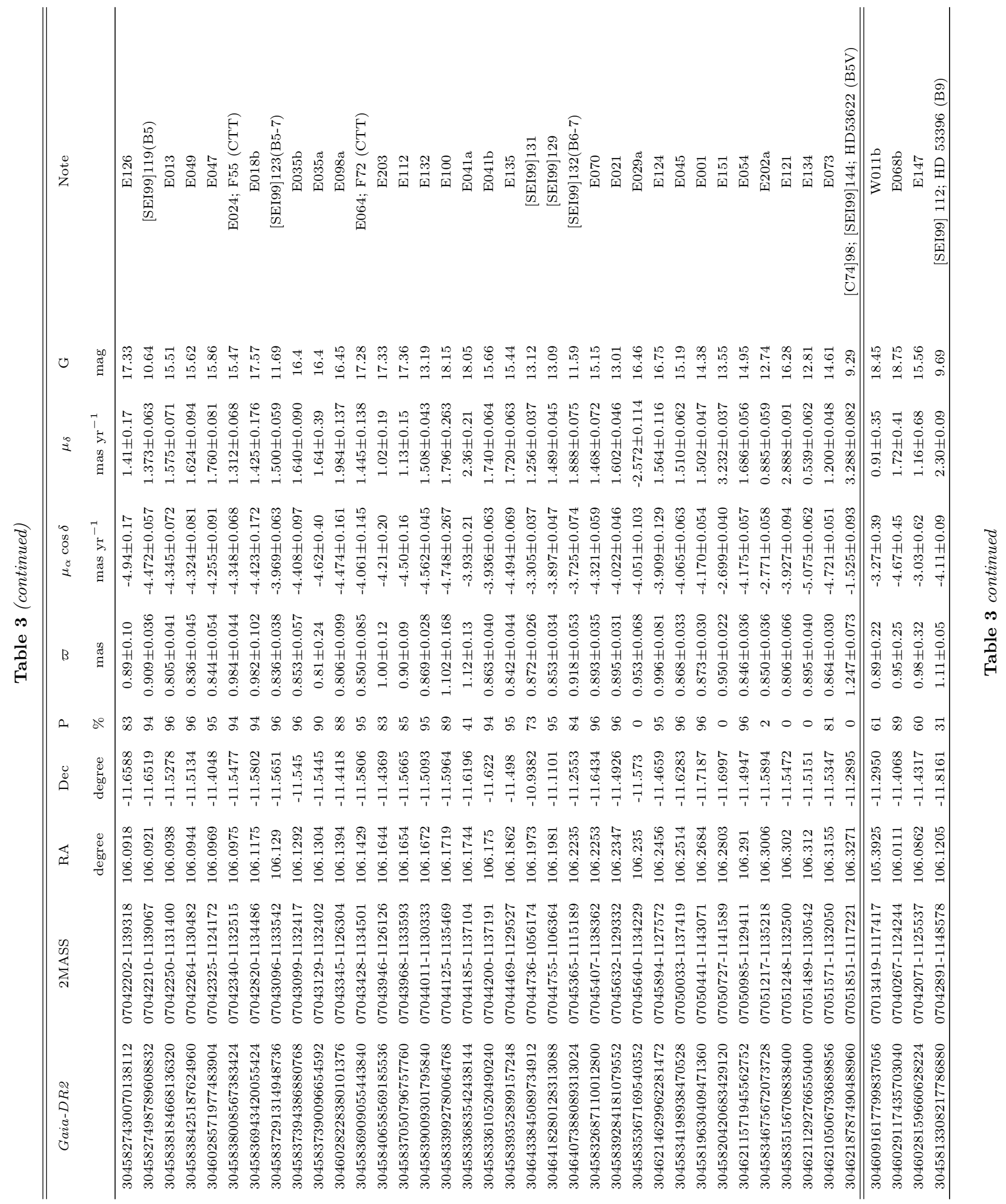




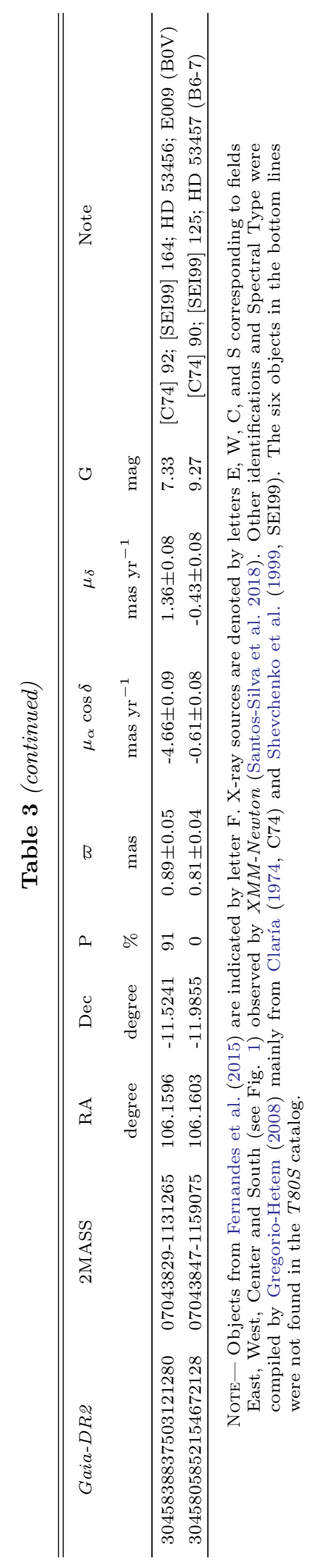




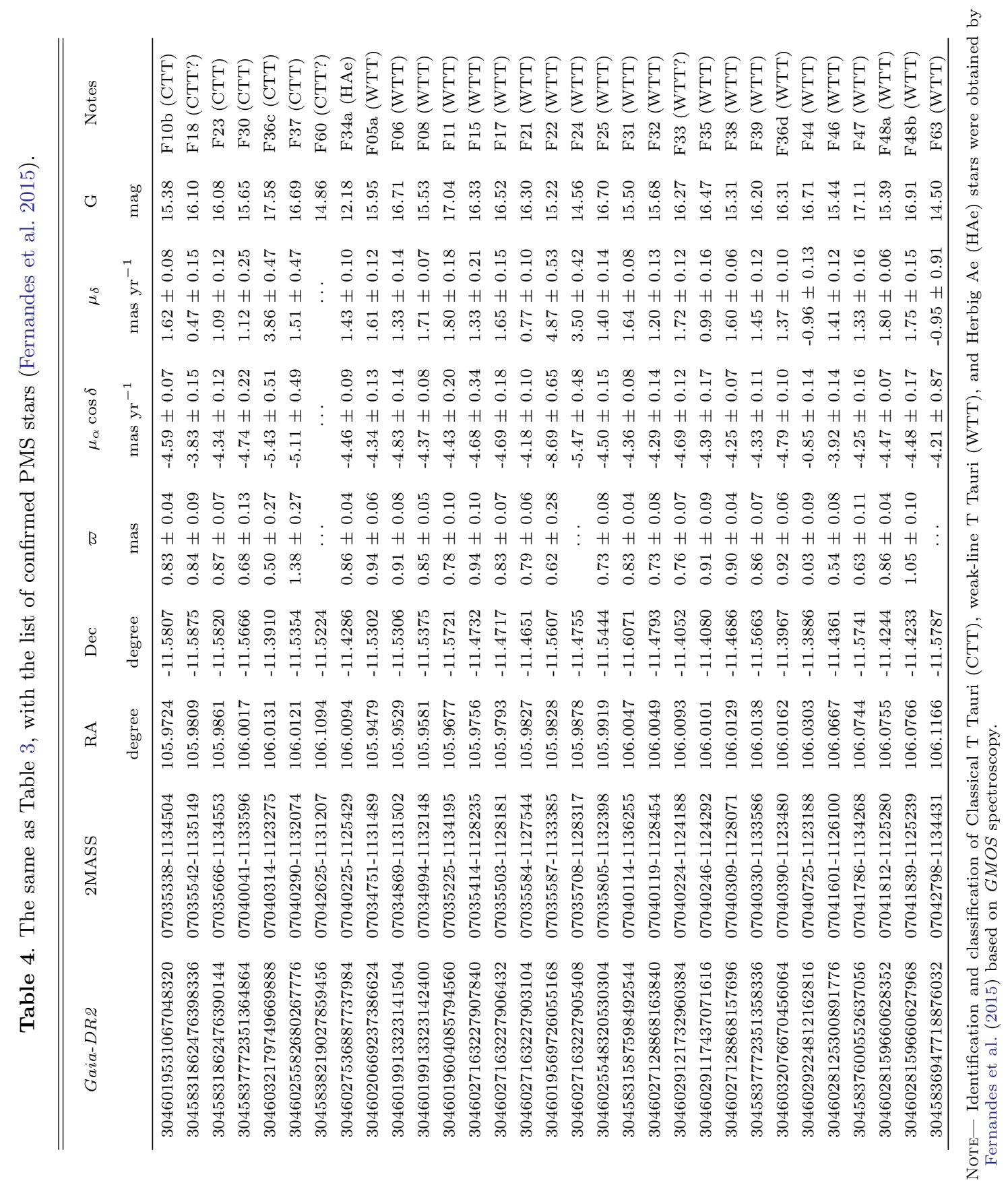


Table 5. New members of CMa R1 identified in the present work. The astrometric data are the same as Table 3 .

\begin{tabular}{|c|c|c|c|c|c|c|c|c|}
\hline Gaia-DR2 & 2MASS & RA & Dec & $\mathrm{P}$ & $\varpi$ & $\mu_{\alpha} \cos \delta$ & $\mu_{\delta}$ & $\mathrm{G}$ \\
\hline & & degree & degree & $\%$ & mas & mas $\mathrm{yr}^{-1}$ & $\operatorname{mas} \mathrm{yr}^{-1}$ & mag. \\
\hline 3046107977332044032 & 07011780-1107209 & 105.3242 & -11.1225 & 70 & $1.10 \pm 0.02$ & $-5.02 \pm 0.04$ & $1.94 \pm 0.04$ & 13.79 \\
\hline 3046013792988609408 & 07012500-1132538 & 105.3542 & -11.5483 & 84 & $0.83 \pm 0.16$ & $-4.69 \pm 0.24$ & $2.05 \pm 0.24$ & 18.05 \\
\hline . & $\cdot$ & $\cdot$ & $\cdot$ & $\cdot$ & $\cdot$ & $\cdot$ & $\cdot$ & $\cdot$ \\
\hline . & . & . & . & . & . & . & . & . \\
\hline$\cdot$ & $\cdot$ & . & $\cdot$ & $\cdot$ & $\cdot$ & $\cdot$ & $\cdot$ & $\cdot$ \\
\hline 3046250295370429824 & $07063757-1108473$ & 106.6566 & -11.1465 & 57 & $1.07 \pm 0.13$ & $-3.22 \pm 0.22$ & $1.83 \pm 0.23$ & 17.80 \\
\hline 3046145150268232960 & 07063763-1142490 & 106.6569 & -11.7136 & 78 & $0.96 \pm 0.28$ & $-3.30 \pm 0.38$ & $1.33 \pm 0.35$ & 18.23 \\
\hline
\end{tabular}

Note- For illustration, only a part of the table is presented. The entire list of objects is available on-line.

Table 6. New candidates $(\mathrm{P}<50$ percent) identified in the present work. The astrometric data are the same as Table 3 .

\begin{tabular}{|c|c|c|c|c|c|c|c|c|}
\hline Gaia-DR2 & 2MASS & $\begin{array}{c}\text { RA } \\
\text { degree }\end{array}$ & $\begin{array}{c}\text { Dec } \\
\text { degree }\end{array}$ & $\begin{array}{l}\mathrm{P} \\
\%\end{array}$ & $\begin{array}{c}\varpi \\
\operatorname{mas}\end{array}$ & $\begin{array}{c}\mu_{\alpha} \cos \delta \\
\operatorname{mas} \mathrm{yr}^{-1}\end{array}$ & $\begin{array}{c}\mu_{\delta} \\
\operatorname{mas} \mathrm{yr}^{-1}\end{array}$ & $\begin{array}{c}\mathrm{G} \\
\mathrm{mag} .\end{array}$ \\
\hline 3046092820387743360 & 07011607-1115513 & 105.3170 & -11.2643 & 34 & $0.96 \pm 0.28$ & $-3.37 \pm 0.50$ & $0.43 \pm 0.44$ & 18.78 \\
\hline 3046067879517494272 & 07011974-1121119 & 105.3323 & -11.3533 & 2 & $0.86 \pm 0.11$ & $-3.34 \pm 0.19$ & $0.39 \pm 0.20$ & 16.88 \\
\hline$\cdot$ & . & $\cdot$ & $\cdot$ & . & $\cdot$ & $\cdot$ & $\cdot$ & . \\
\hline · & . & $\cdot$ & · & · & · & $\cdot$ & · & · \\
\hline$\cdot$ & $\cdot$ & $\cdot$ & $\cdot$ & $\cdot$ & $\cdot$ & $\cdot$ & $\cdot$ & $\cdot$ \\
\hline 3046441507311707392 & 07062918-1058194 & 106.6216 & -10.9720 & 20 & $1.06 \pm 0.12$ & $-2.81 \pm 0.19$ & $1.20 \pm 0.21$ & 17.27 \\
\hline 3046448821637725952 & 07063299-1054190 & 106.6375 & -10.9053 & 3 & $0.89 \pm 0.04$ & $-4.38 \pm 0.06$ & $2.53 \pm 0.07$ & 12.78 \\
\hline
\end{tabular}

Note- For illustration, only a part of the table is presented. The entire list of objects is available on-line. 
SEARCHING FOR ACTIVE LOW-MASS STARS IN CMA REGION

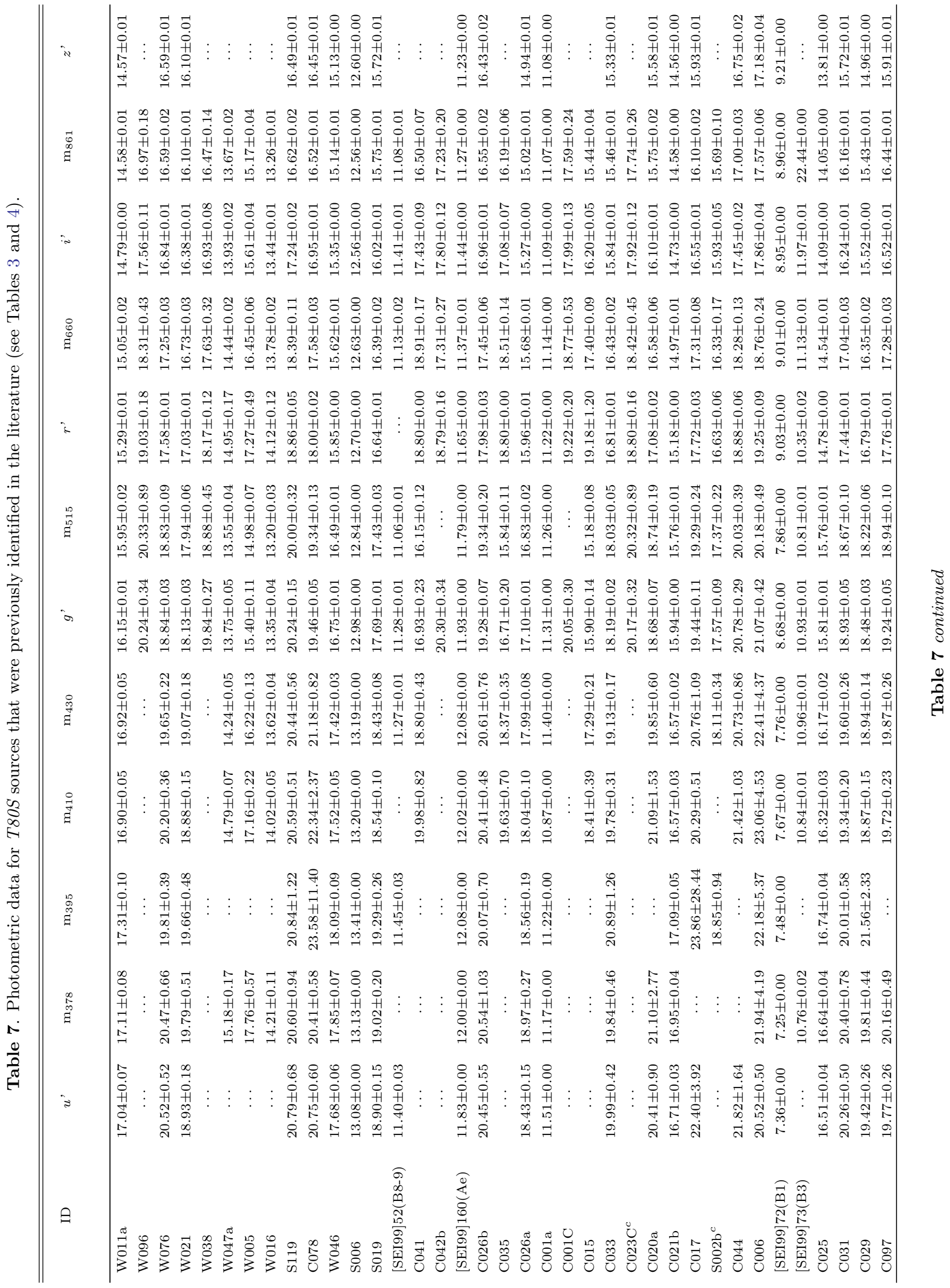




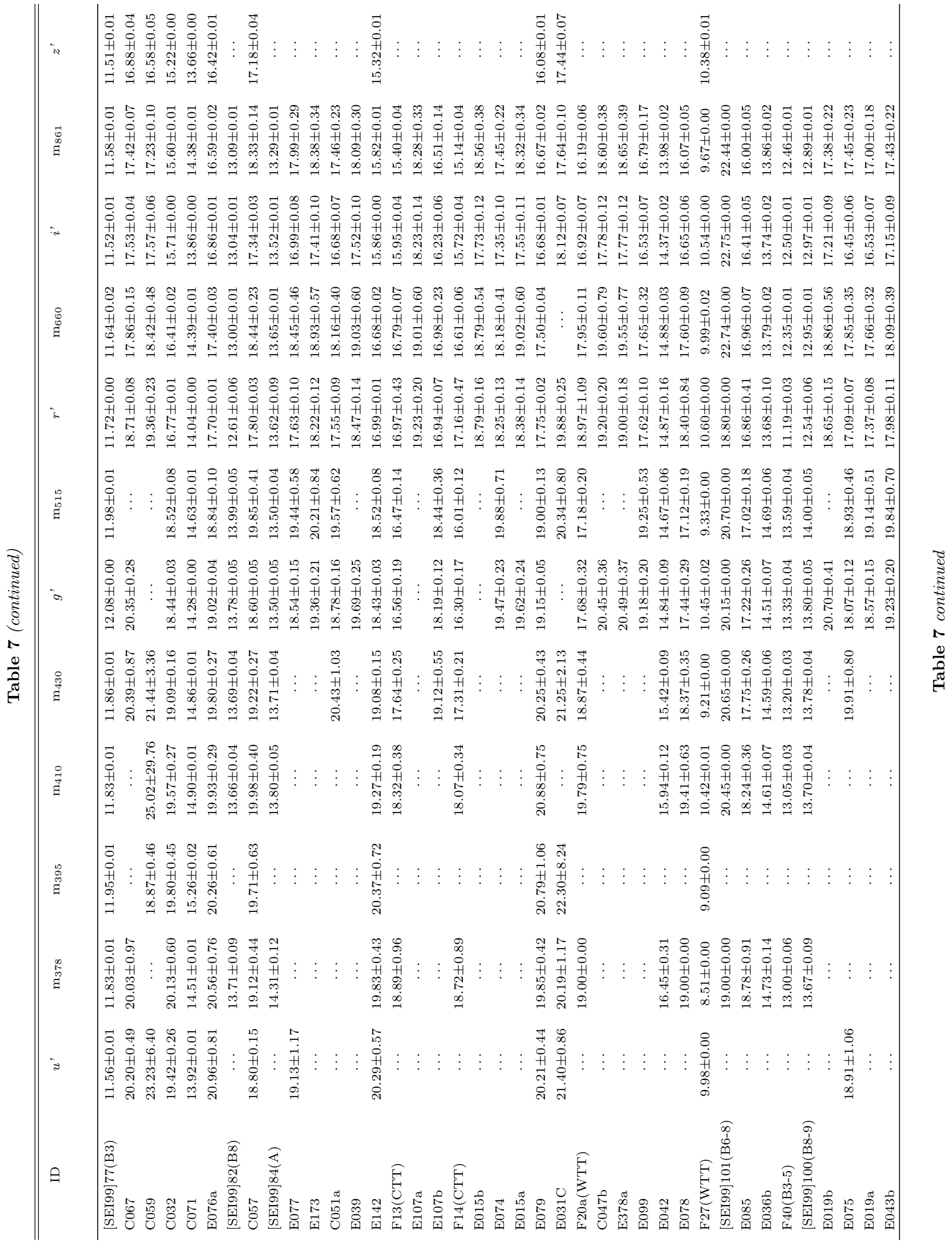




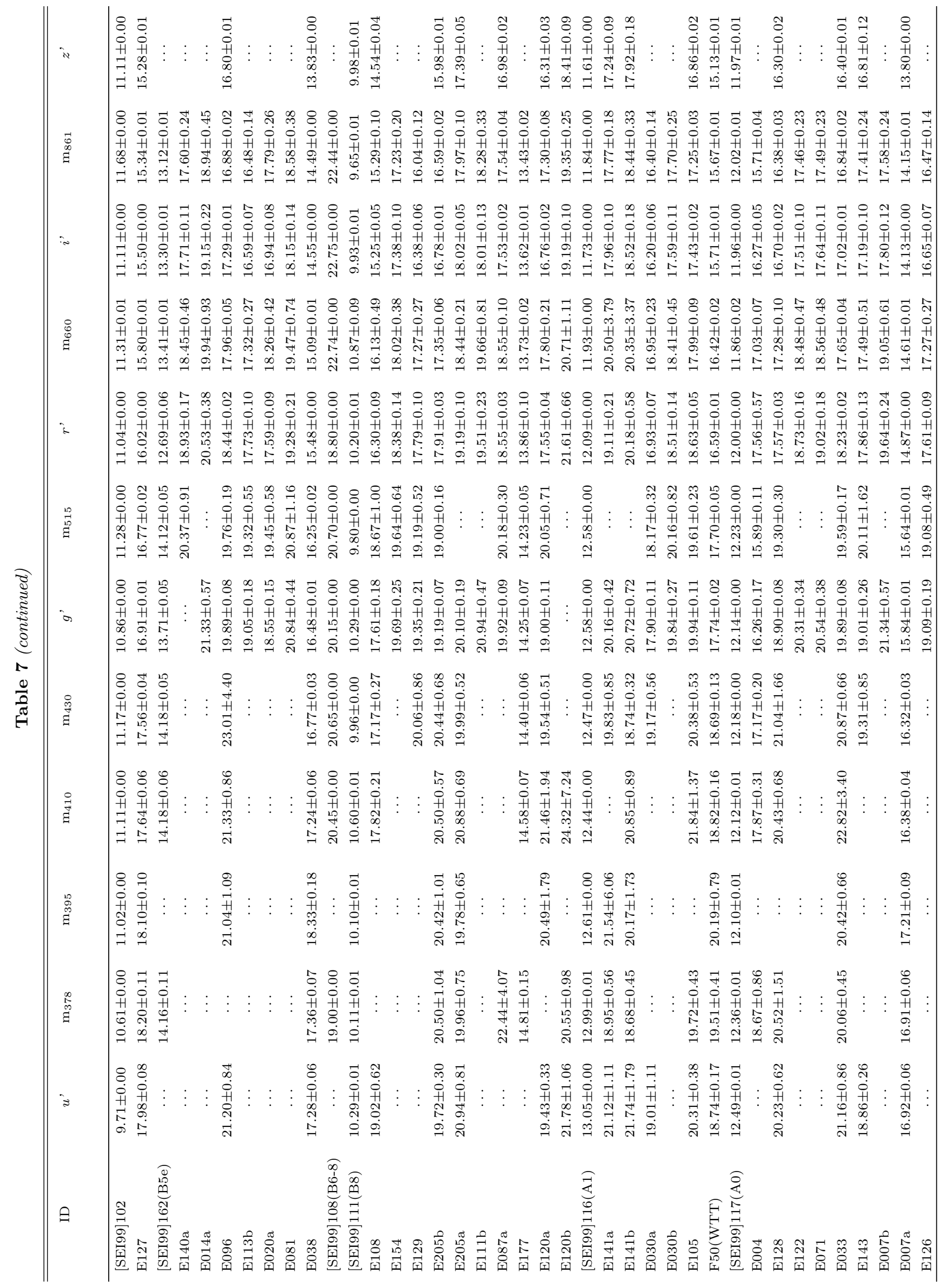




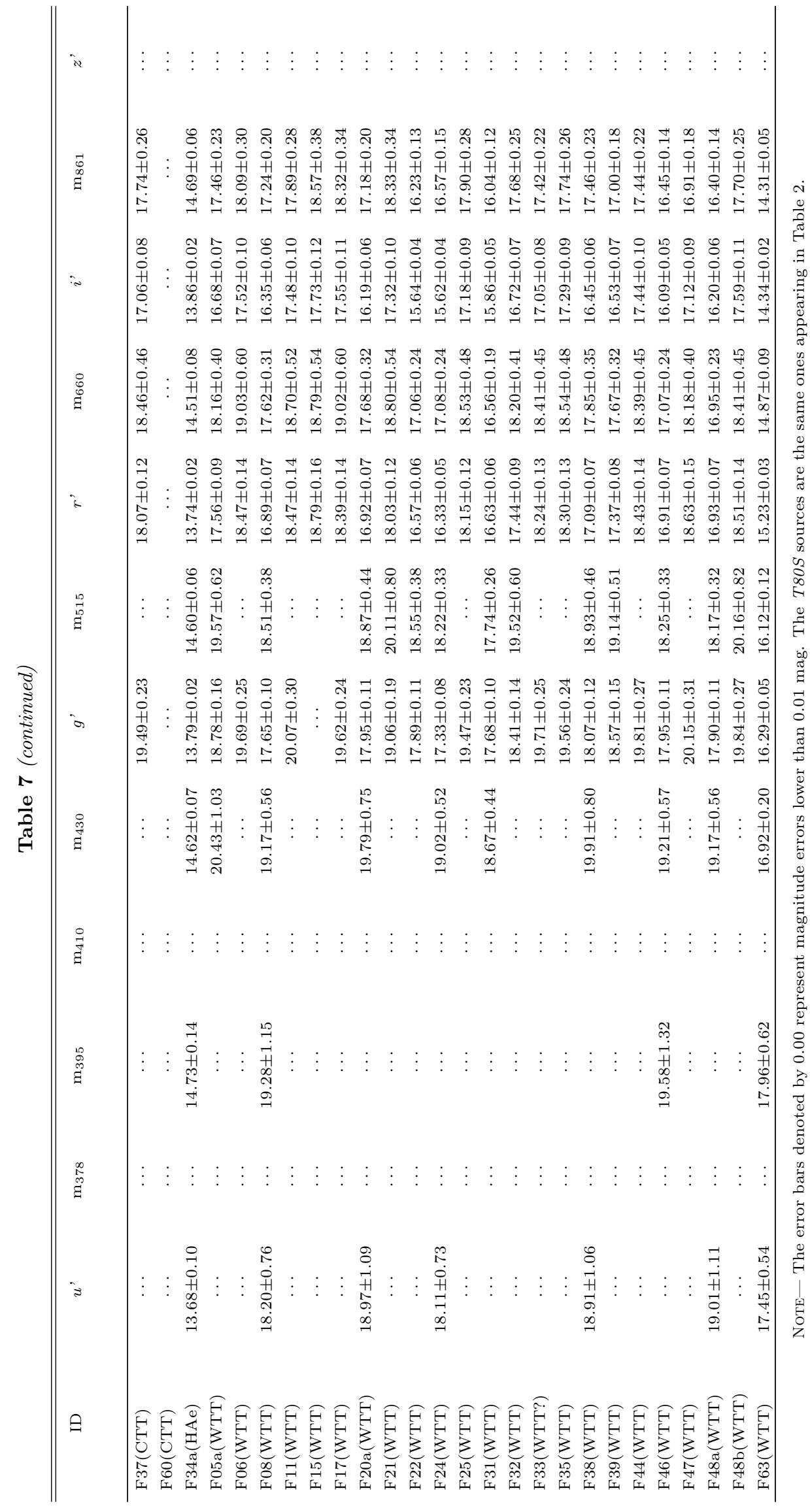




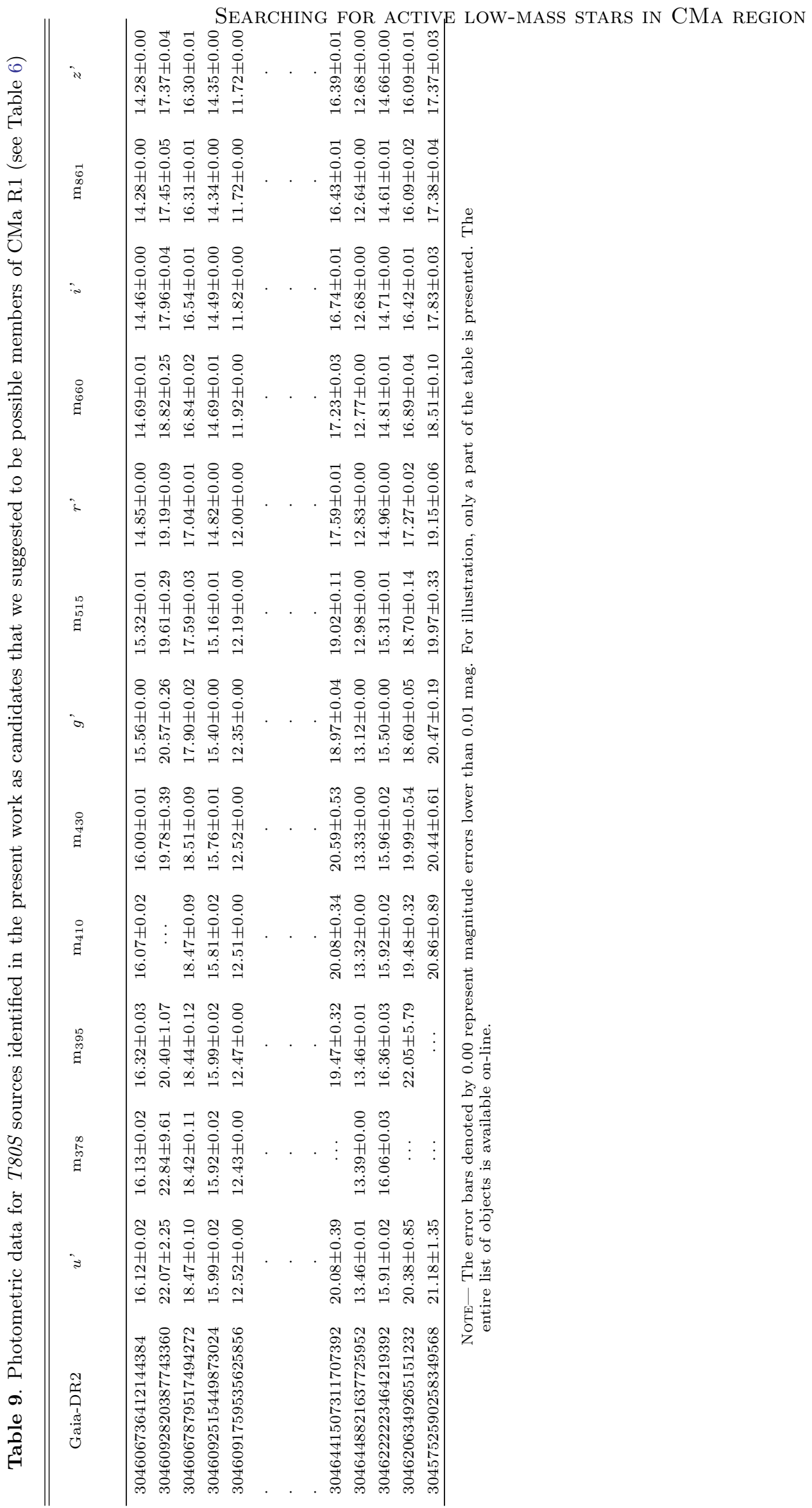




\section{ACKNOWLEDGMENTS}

This work was partially supported by FAPESP Proc. 2014/18100-4 (JGH), Proc. 2017/18191-8 (FN), Proc. 2017/19458-8 (AH), Proc. 2018/06822-6 (TSS), Proc. 2018/21250-9 (HDP), Proc. 2018/05866-0 (VJP). JGH acknowledges support from CNPq (305590/2016-6). We thank Stavros Akras, Fábio R. Herpich, and Alvaro Alvarez-Candal for their suggestions and comments on the manuscript.

This work has made use of data from the European Space Agency (ESA) mission Gaia (https://www. cosmos.esa.int/gaia), processed by the Gaia Data Processing and Analysis Consortium (DPAC, https://www. cosmos.esa.int/web/gaia/dpac/consortium). Funding for the DPAC has been provided by national institutions, in particular the institutions participating in the Gaia Multilateral Agreement.

The S-PLUS project, including the T80-South robotic telescope and the S-PLUS scientific survey, was founded as a partnership between the Fundação de Amparo à Pesquisa do Estado de São Paulo (FAPESP), the Observatório Nacional (ON), the Federal University of Sergipe (UFS), and the Federal University of Santa Catarina
(UFSC), with important financial and practical contributions from other collaborating institutes in Brazil, Chile (Universidad de La Serena), and Spain (Centro de Estudios de Física del Cosmos de Aragón, CEFCA). We further acknowledge financial support from the São Paulo Research Foundation (FAPESP), the Brazilian National Research Council (CNPq), the Coordination for the Improvement of Higher Education Personnel (CAPES), the Carlos Chagas Filho Rio de Janeiro State Research Foundation (FAPERJ), and the Brazilian Innovation Agency (FINEP).

The authors who are members of the S-PLUS collaboration are grateful for the contributions from CTIO staff in helping in the construction, commissioning and maintenance of the T80-South telescope and camera. We are also indebted to Rene Laporte and INPE, as well as Keith Taylor, for their important contributions to the project. From CEFCA, we thank Antonio Marín-Franch for his invaluable contributions in the early phases of the project, David Cristóbal-Hornillos and his team for their help with the installation of the data reduction package JYPE version 0.9.9, César Íñiguez for providing 2D measurements of the filter transmissions, and all other staff members for their support with various aspects of the project.

\section{REFERENCES}

André, Ph., Men'shchikov, A., Bontemps, S. et al. 2010, A\&A, 518, 102

Bessell, M. S., 1990, PASP, 102, 1181

Bertin, E. 2010, SWarp: Resampling and Co-adding FITS Images Together, Astrophysics Source Code Library (ascl:1010.068)

Bertin E., Arnouts S., 1996, A\&A, 117, 393

Bonatto, C. 2019, MNRAS, 483, 2758

Brand, J., Massi, F., Zavagno, A., Deharveng, L., Lefloch, B. 2011, A\&A, 527,62

Bressan, A., Marigo, P., Girardi, L., Salasnich, B., Dal Cero, C., Rubele, S., Nanni, A., 2012, MNRAS, 427, 127

Briceño, C., Calvet, N., Hernández, J. et al. 2019, AJ, 157, 85

Cenarro, A. J., et al. 2019, A\&A, 622, A176

Christiaens, V.; Cantalloube, F.; Casassus, S.; Price, D.J.;

Absil, O.; Pinte, C.; Girard, J.; Montesinos, M., 2019, ApJ, 877, L33

Claría, J. J., 1974, AJ, 79, 1022

Cloutier, R., Currie, T., Rieke, G. H., et al. 2014, ApJ, 796, 127

Cohen, M., Kuhi, L. V. 1979 ApJS41, 743
Covey, K. R., Ivezić, Ž., Schlegel, D. et al. 2007, AJ, 134, 2398

Diolaiti, E., Bendinelli, O., Bonaccini, D., Close, L., Currie, D., Parmeggiani, G., 2000, A\&AS, 147, 335

Donati, J.-F., Skelly, M. B., Bouvier, J. et al., 2010 , MNRAS, 402, 1426

Drew, J. E., Gonzalez-Solares, E., Greimel, R. , et al., 2014, MNRAS, 440, 2036

Fang, M., van Boekel, R., Wang, W., et al., 2009, A\&A, 504,461

Fedele, D., van den Ancker, M. E., Henning, Th., Jayawardhana, R., Oliveira, J. M., 2010, A\&A, 510, 72

Feigelson, E. D., Montmerle, T., 1999 ARA\&A, 37, 363

Fernandes, B., Gregorio-Hetem, J., Montmerle, T., Rojas, G., 2015, MNRAS, 448, 119

Fernandes, B.; Montmerle, T., Gregorio-Hetem, J., Santos-Silva, T., 2019 A\&A, 628, 44

Fischer, W. J.; Padgett, D. L., Stapelfeldt, K. L., Sewilo, M., 2016, ApJ, 827, 96

Gaia Collaboration, Brown, A. G. A., Vallenari, A., Prusti, T. et al. 2018a A\&A, 616, 1

Gaia Collaboration, Babusiaux, C., van Leeuwen, F., Barstow, M. A. et al. 2018b, A\&A, 616, A10 
Galli, P. A. B., Bertout, C., Teixeira, R., Ducourant, C., 2013, A\&A, 558, 77

Gregorio-Hetem, J., Lépine, J. R. D., Quast, G. R., Torres, C. A. O., de La Reza, R., 1992, AJ, 103, 549

Gregorio-Hetem, J.; Hetem, A. 2002, MNRAS, 336, 197

Gregorio-Hetem, J. 2008, Handbook of Star Forming Regions, Vol. II, p. 1

Gregorio-Hetem, J., Montmerle, T., Rodrigues, C. V. et al., 2009, A\&A, 506, 711

Gregorio-Hetem, J., Hetem, A., Santos-Silva, T., Fernandes, B., 2015, MNRAS, 448, 2504

Gregorio-Hetem, J., Fernandes, B., Santos-Silva, T., Montmerle, T., 2016, in "Interstellar Clouds to Star-forming Galaxies: Universal Processes?", Proceedings IAU Symposium No. 315, P. Jablonka, Ph. André \& F. Van der Tak, eds.

Haisch, K. E., Lada, E. A., \& Lada, C. J., 2001, ApJ, 553, L153

Hashimoto, J., Tsukagoshi, T., Brown, J. M. et al. 2015, ApJ, 799, 43

Hashimoto, J., Dong, R., Kudo, T. et al. 2012, ApJ758, 19

Hernández, J., Hartmann, L., Calvet, N., et al. 2008, ApJ, 686, 1195

Hetem, A. \& Gregorio-Hetem, J., 2007, MNRAS, 382, 1707

Hetem, A., Gregorio-Hetem, J., 2019, MNRAS, 490, 2521

Kalari, V. M., Vink, J. S., Drew, J. E., et al., 2015, MNRAS, 453, 1026

Keppler, M., Benisty, M., Müller, A., et al. 2018, A\&A, 617, A44

Koenig, X. P., Leisawitz, D. T. 2014, ApJ, 791, 131
Maíz Apellániz, J., 2006, AJ, 131, 1184

Marigo P. et al., 2017, ApJ, 835, 77

Martínez-Arnáiz R.; López-Santiago, J.; Crespo-Chacón, I.; Montes, D., 2011 MNRAS, 414, 2629

Mendes de Oliveira, C., Ribeiro, T., Schoenell, W., et al., 2019, MNRAS, 489, 241

Molino A., Benítez, N., Moles, M. et al., 2014, MNRAS, 441,2891

Müller, A., Keppler, M., Henning, Th. et al. 2018, A\&A, 617, L2

Onishi, T., Nishimura, A., Ota, Y., et al. 2013, PASJ, 65, 78

Preibisch, T., 2012, Resarch in Astronomy and Astrophysics, 12, 1

Rebull, L. M., Padgett, D. L., McCabe, C.-E. et al., 2010, ApJS, 186, 259

Riaud, P.; Mawet, D.; Absil, O.; Boccaletti, A.; Baudoz, P.; Herwats, E.; Surdej, J., 2006, A\&A, 458, 317

Santos-Silva, T., Perottoni, H.D., Almeida-Fernandes, F. et al. , 2020 A\&A, (in preparation)

Santos-Silva, T.; Gregorio-Hetem, J.; Montmerle, T.; Fernandes, B.; Stelzer, B., 2018 A\&A, 609, 127

Shevchenko, V. S., Ezhkova, O. V., Ibrahimov, M. A. et al. 1999, MNRAS, 310, 210

Siess L., Dufour E., Forestini M., 2000, A\&A, 358, 593

Venuti, L., Bouvier, J., Flaccomio, E., Alencar, S. H. P., et al. 2014, A\&A, 570, 82

Wiramihardja et al., 1986, PASJ38, 395

Wright, E. L., Eisenhardt, P. R. M., Mainzer, A. K., et al. 2010, AJ, 140, 1868 